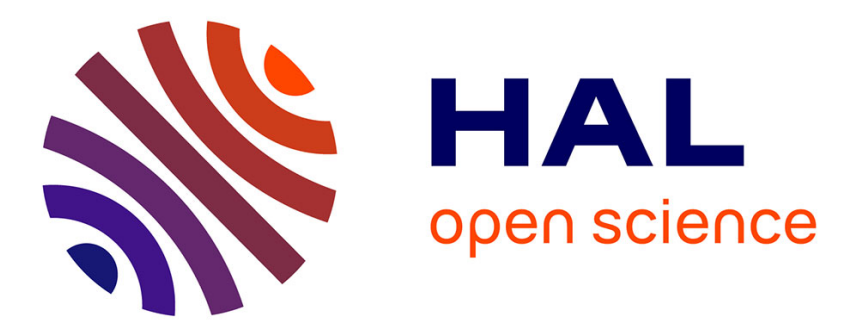

\title{
Structural insights into photosystem II assembly
}

Jure Zabret, Stefan Bohn, Sandra K Schuller, Oliver Arnolds, Madeline

Möller, Jakob Meier-Credo, Pasqual Liauw, Aaron Chan, Emad Tajkhorshid, Julian D Langer, et al.

\section{- To cite this version:}

Jure Zabret, Stefan Bohn, Sandra K Schuller, Oliver Arnolds, Madeline Möller, et al.. Structural insights into photosystem II assembly. Nature Plants, 2021, 7 (4), pp.524-538. 10.1038/s41477-02100895-0 . hal-03406233

\section{HAL Id: hal-03406233 \\ https://hal.science/hal-03406233}

Submitted on 27 Oct 2021

HAL is a multi-disciplinary open access archive for the deposit and dissemination of scientific research documents, whether they are published or not. The documents may come from teaching and research institutions in France or abroad, or from public or private research centers.
L'archive ouverte pluridisciplinaire HAL, est destinée au dépôt et à la diffusion de documents scientifiques de niveau recherche, publiés ou non, émanant des établissements d'enseignement et de recherche français ou étrangers, des laboratoires publics ou privés. 


\section{Structural insights into photosystem II assembly}

Jure Zabret ${ }^{1 \#}$, Stefan Bohn²\#, Sandra K. Schuller ${ }^{3,4}$, Oliver Arnolds ${ }^{5}$, Madeline Möller ${ }^{1}$, Jakob Meier-Credo ${ }^{6}$, Pasqual Liauw ${ }^{1}$, Aaron Chan ${ }^{7}$, Emad Tajkhorshid ${ }^{7}$, Julian D. Langer ${ }^{6,8}$, Raphael Stoll ${ }^{5}$, Anja Krieger-Liszkay ${ }^{9}$, Benjamin D. Engel2,10,11, Till Rudack ${ }^{12,13, *}$, Jan M. Schuller ${ }^{3,4, *}$, Marc M. Nowaczyk ${ }^{1, *}$

\section{Affiliations:}

${ }^{1}$ Department of Plant Biochemistry, Faculty of Biology \& Biotechnology, Ruhr University Bochum, 44780 Bochum, Germany.

${ }^{2}$ Department of Molecular Structural Biology, Max Planck Institute of Biochemistry, 82152 Martinsried, Germany.

${ }^{3}$ Department of Structural Cell Biology, Max Planck Institute of Biochemistry, 82152 Martinsried, Germany.

${ }^{4}$ CryoEM of Molecular Machines, SYNMIKRO Research Center and Department of Chemistry, Philipps University of Marburg, 35043 Marburg, Germany.

${ }^{5}$ Biomolecular Spectroscopy and RUBiospek|NMR, Faculty of Chemistry and Biochemistry, Ruhr-University Bochum, 44780 Bochum, Germany

${ }^{6}$ Department of Molecular Membrane Biology, Max Planck Institute of Biophysics, 60438 Frankfurt/Main, Germany.

${ }^{7} \mathrm{NIH}$ Center for Macromolecular Modeling and Bioinformatics, Beckman Institute for Advanced Science and Technology, Department of Biochemistry, and Center for Biophysics and Quantitative Biology, University of Illinois at Urbana-Champaign, Urbana, Illinois

${ }^{8}$ Max Planck Institute for Brain Research, Max von Laue Strasse 4, 60438 Frankfurt/Main, Germany.

${ }^{9}$ Université Paris-Saclay, CEA, CNRS, Institute for Integrative Biology of the Cell (I2BC), 91198, Gif-sur-Yvette, France

${ }^{10}$ Helmholtz Pioneer Campus, Helmholtz Zentrum München, Ingolstädter Landstraße 1, 85764 Neuherberg, Germany.

${ }^{11}$ Department of Chemistry, Technical University of Munich, Lichtenbergstraße 4, 85748 Garching, Germany.

${ }^{12}$ Biospectroscopy, Center for Protein Diagnostics (ProDi), Ruhr University Bochum, 44801 Bochum, Germany.

${ }^{13}$ Department of Biophysics, Faculty of Biology \& Biotechnology, Ruhr University Bochum, 44780 Bochum, Germany.

\#Equal contribution

*corresponding authors: till.rudack@,rub.de, jan.schuller@synmikro.uni-marburg.de, marc.m.nowaczyk@rub.de 


\title{
Keywords
}

photosynthesis, photosystem II biogenesis, assembly factors, bicarbonate binding, reactive oxygen species, protection mechanisms, oxygen evolving complex, photoactivation, cryo-EM

\begin{abstract}
Biogenesis of photosystem II (PSII), nature's water splitting catalyst, is assisted by auxiliary proteins that form transient complexes with PSII components to facilitate stepwise assembly events. Using cryo-electron microscopy, we solved the structure of such a PSII assembly intermediate from Thermosynechococcus elongatus at $2.94 \AA$ resolution. It contains three assembly factors (Psb27, Psb28, Psb34) and provides detailed insights into their molecular function. Binding of Psb28 induces large conformational changes at the PSII acceptor side, which distort the binding pocket of the mobile quinone $\left(\mathrm{Q}_{\mathrm{B}}\right)$ and replace the bicarbonate ligand of non-heme iron with glutamate, a structural motif found in reaction centers of non-oxygenic photosynthetic bacteria. These results reveal novel mechanisms that protect PSII from damage during biogenesis until water splitting is activated. Our structure further demonstrates how the PSII active site is prepared for the incorporation of the $\mathrm{Mn}_{4} \mathrm{CaO}_{5}$ cluster, which performs the unique water splitting reaction.
\end{abstract}

\section{Introduction}

2 Photosystem II (PSII) is the only enzyme that catalyzes the light-driven oxidation of water, a 3 thermodynamically demanding reaction that drives photosynthesis, sustaining life on our 4 planet $^{1-3}$. This multi-subunit membrane protein complex is located in the thylakoid membranes of cyanobacteria, algae and plants. PSII strips electrons from water and injects them into the photosynthetic electron transport chain (PET). It forms a homodimer with a molecular mass of $\sim 500 \mathrm{kDa}^{4}$, with each monomer composed of at least 20 protein subunits and numerous cofactors, including chlorophylls, quinones, carotenoids, lipids, bicarbonate and the unique $\mathrm{Mn}_{4} \mathrm{CaO}_{5}$ cluster $^{5-7}$. The two core proteins D1 and D2 form a central, membrane-intrinsic heterodimer, which binds all important redox cofactors involved in internal electron transfer ${ }^{8}$. Light-excitation leads to a charge-separated state in which an electron is transferred from the reaction center chlorophylls $\mathrm{P}_{680}{ }^{9}$ to the nearby pheophytin ${ }^{10}$. Subsequently, the electron is passed to the bound plastoquinone $\left(\mathrm{Q}_{\mathrm{A}}\right)$ and then to the mobile plastoquinone molecule $\left(\mathrm{Q}_{\mathrm{B}}\right)$, which leaves the complex after accepting two electrons and two protons ${ }^{11}$. The electron hole at $\mathrm{P}_{680}$ is filled by oxidation of an adjacent tyrosine residue $\left(\mathrm{Tyr}_{\mathrm{Z}}\right)^{12}$ and finally by the oxygen 
evolving complex (OEC), which contains the $\mathrm{Mn}_{4} \mathrm{CaO}_{5}$ cluster. In cyanobacteria, the cluster is shielded on the luminal side by the three extrinsic proteins, PsbO, PsbU and PsbV, which regulate access to the OEC by forming an intricate network of channels for different substrates and products ${ }^{13}$. Light energy is collected and funneled towards $\mathrm{P}_{680}$ by the two membraneintrinsic antenna proteins CP43 and CP47. These proteins bind most of the chlorophyll molecules and are located on opposite sides of the D1/D2 heterodimer ${ }^{14}$. Moreover, at least twelve small transmembrane subunits with one or two transmembrane helices have been identified in PSII ${ }^{15}$, including cytochrome- $\mathrm{b}_{559}{ }^{16}$.

Structural and spectroscopic investigations have revealed these aforementioned comprehensive insights into PSII function ${ }^{17-21}$, but we are far from understanding PSII biogenesis with molecular detail. How nature facilitates the assembly of a multi-subunit, multi-cofactor membrane protein complex is a fundamental unsolved question. The biogenesis of PSII is even more challenging, as the mature complex performs sophisticated and extreme redox chemistry to catalyze the light-driven oxidation of water. This can easily lead to the formation of reactive oxygen species (e.g., singlet oxygen is produced by triplet chlorophyll in the PSII reaction center) and subsequent loss of function due to damaged proteins and cofactors ${ }^{22,23}$. Biogenesis intermediates with only partially functional fragments of the redox chain are particularly prone to damage, thus demanding specialized protection mechanisms for the assembly process ${ }^{24}$. Therefore, PSII biogenesis is not a spontaneous process but rather must be tightly regulated by the action of assembly factors. Thus far, more than 20 auxiliary proteins have been identified that guide the stepwise assembly of PSII subunits and cofactors via intermediate modules, which are assembled independently and then joined together to produce mature PSII ${ }^{25-27}$. In cyanobacteria, PSII biogenesis begins with the formation of the D1/D2 heterodimer reaction center $(\mathrm{RC})$ complex from the $\mathrm{D} 1$ precursor protein $(\mathrm{pD} 1)$ and the $\mathrm{D} 2$ protein. This is assisted by the PSII assembly factor Ycf48 after partial processing of the D1 C-terminal extension by the D1 specific peptidase CtpA ${ }^{28,29}$. In the next step, the assembly factor Psb28 helps CP47 join the RC complex to form the RC47 complex, in which iD1 is further processed to its mature form by $\mathrm{CtpA}^{30,31}$. Almost all ligands of the $\mathrm{Mn}_{4} \mathrm{CaO}_{5}$ cluster are already present at this stage, except for those provided by CP43, which comes pre-constructed with assembly factor Psb27 and several small subunits (together called the CP43 module) ${ }^{32}$. Psb28 is released as CP43 binds, and the resulting Psb27-PSII monomer is activated by maturation of the OEC and the binding of the extrinsic proteins PsbO, PsbU and PsbV ${ }^{33-35}$. OEC assembly is a multistep process that requires a functional upstream redox chain for the oxidation of $\mathrm{Mn}^{2+}$ to build up the cluster's $\mu$-oxo bridges between the manganese atoms ${ }^{36,37}$. The mechanistic and structural 
details of this photoactivation process are not yet understood. In the consensus 'two quantum model ${ }^{38-40}$, a single $\mathrm{Mn}^{2+}$ ion bound to the high-affinity site (HAS) ${ }^{41}$ is oxidized to $\mathrm{Mn}^{3+}$. This initiating light-dependent step is followed by a slow light-independent phase and further fast light-dependent steps in which the remaining $\mathrm{Mn}^{2+}$ ions are oxidized and incorporated. Understanding the light-independent slow phase is key to unraveling the mechanism of photoactivation. Finally, PSII biogenesis completes with dimerization of two fully assembled monomers and attachment of the soluble phycobilisome antenna complexes. Interestingly, deletion of $p s b J$, which encodes a small single transmembrane helix protein at the entrance of the PSII plastoquinone channel, decreases PSII-mediated oxygen evolution and increases the lifetime of the reduced primary acceptor $\left(\mathrm{Q}_{\mathrm{A}}^{-}\right)$in cyanobacterial and tobacco chloroplasts ${ }^{42}$. These effects are caused by a massive accumulation of an intermediate monomeric PSII complex, which contains both assembly factors Psb27 and Psb28 $8^{43}$. Physiological studies of Psb27 and Psb28 deletion strains point towards multifaceted functions. Cyanobacterial mutants lacking Psb28 exhibited slower autotrophic growth, particularly under stress conditions ${ }^{31,44}$, and limited synthesis of Chl-binding proteins but without decreased PSII functionality ${ }^{31}$. The Psb28 mutant also exhibited an overall increase in PSII repair and faster recovery from photodamage ${ }^{31}$. Chemical cross-linking combined with mass spectrometry revealed that Psb28 binds to the cytosolic side of CP47 close to cytochrome- $\mathrm{b}_{559}$ and the $\mathrm{Q}_{\mathrm{B}}$ binding site. Based on this, researchers postulated a protective role for Psb28, where it blocks electron transport to the acceptor side of PSII to shield the RC47 complex from excess photodamage during the assembly process ${ }^{45}$. This hypothesis is strengthened by the observation that Psb28 is also found in PSII repair complexes ${ }^{46}$. The luminal PSII assembly factor Psb27 has been similarly well investigated. This lipoprotein is predominantly associated with inactive PSII fractions involved in assembly or repair ${ }^{32,34,46-50}$, stabilizing the CP43 luminal domain and presumably facilitating the assembly of the OEC.

Our current knowledge of PSII biogenesis mainly describes the order of events and protein composition of each intermediate, as well as the general roles of PSII assembly factors. However, the precise molecular functions of these intermediate complexes and the involved assembly factors are still elusive due to their low abundance and intrinsic instability. Highresolution structural information is of vital importance to gain a deeper understanding into the molecular action of PSII assembly factors, as they are proposed to alter the structures of their associated PSII proteins to provide protection or facilitate specific biogenesis transitions.

Here, we use cryo-EM single particle analysis to describe the first molecular structure of a PSII assembly intermediate. This structure represents one of the key transitions in PSII biogenesis: 
the attachment of the CP43 module to the pre-assembled RC47 reaction center complex, which precedes incorporation and activation of the $\mathrm{Mn}_{4} \mathrm{CaO}_{5}$ cluster. We complement this structural data with spectroscopic analysis, revealing the first detailed insights into the molecular mechanisms of PSII assembly. Our study provides mechanistic answers to three long-standing questions: i) How do assembly factors modulate the structures of PSII subunits to assist biogenesis? ii) How is PSII protected from photodamage during assembly? iii) How is the PSII active site prepared for incorporation of the $\mathrm{Mn}_{4} \mathrm{CaO}_{5}$ cluster?

\section{Results}

Stable PSII intermediates were purified from the T. elongatus $\Delta p s b J$ mutant ${ }^{43}$ by affinity chromatography using a twin-strep-tag fused to the C-terminus of the CP43 subunit and subsequent ion exchange chromatography (Fig. S1a). The main peak of the IEC profile corresponds primarily to monomeric PSII, which lacks the extrinsic subunits PsbO, PsbU and PsbV that are indicative for water splitting activity (Fig. S1b and c). Single particle cryo-EM analysis of this PSII fraction resulted in three different high-resolution maps that allowed model building with high confidence and excellent statistics (Fig. S2, Table S1). In addition to the protein subunits, we also faithfully assigned all essential non-protein cofactors, including chlorophylls, quinones, carotenoids and lipids, which are also present in the mature PSII complex (Fig. S3). Consistent with previous biochemical studies ${ }^{34,43,48}$, the EM density corresponding to the fully assembled, active $\mathrm{Mn}_{4} \mathrm{CaO}_{5}$ cluster is missing in the purified biogenesis intermediates. The first cryo-EM map (2.94 $\AA$ ), which we call PSII-I (for PSIIIntermediate), provides a snapshot of the attachment of the CP43 module to the pre-assembled RC47 reaction center complex (Fig. 1). This PSII intermediate contains three assembly factors (Psb27, Psb28 and Psb34), as well as almost all the membrane-intrinsic subunits and cofactors found in mature PSII, although CP43 is bound in an immature conformation. Psb27 and Psb28 are well-known assembly factors ${ }^{31,32,35,43,51}$, whereas the additional single transmembrane helix protein (ts10063), which we named Psb34, has not been described before. The small subunit PsbY, which is known to be loosely bound ${ }^{52}$, is not resolved in our structure. In addition, PsbJ is not present, as the corresponding gene was inactivated to stall PSII assembly at this specific transition $^{43}$.

The two additional maps serve as internal controls. PSII-I' (2.76 $\AA$ ) lacks Psb27 but is otherwise comparable to PSII-I; the root mean square deviation (RMSD) of the $\mathrm{C}_{\alpha}$ atomic positions between similar subunits of the two complexes is $0.4 \AA$. Most likely, Psb27 was partly lost 
during sample preparation. The third cryo-EM map (2.82 $\AA$ ), which we call PSII-M (for PSIIMonomer), represents a monomeric PSII complex without bound assembly factors. Comparison of our PSII-M structure with a crystal structure of monomeric PSII ${ }^{52}$ (PDB-ID 3KZI, $3.6 \AA$ ) reveals only minimal differences between both structures, with a C $\alpha$ RMSD of $1.3 \AA$, which verifies that the structural changes observed in PSII-I are not caused by the deletion of PsbJ.

\section{Psb34 binds to CP47 and is involved in the RC47 transition}

Our PSII-I structure provides the first identification of the single transmembrane helix protein Psb34 bound to a PSII assembly intermediate (Fig. 2a), which we also confirmed by mass spectrometry (Fig. 2b). Psb34 was probably overlooked previously due to its hydrophobicity and small size. It has a single transmembrane helix that binds to the CP47 antenna protein in close proximity to PsbH (Fig. 2a). Its conserved long N-terminal arm is located at the side and top of the D2 subunit (Fig. 2a). In addition, we independently confirmed the interaction of Psb34 with PSII assembly intermediates by isolation of strep-tagged Psb34 complexes from otherwise unmodified cells, indicating a specific function of Psb34 in the attachment of CP43 to RC47 (Fig. 2c). In this case, two distinct PSII intermediates were isolated via pulldown of strep-tagged Psb34: the RC47 complex with bound Psb28 and the subsequent PSII intermediate after attachment of CP43 and Psb27 (Fig. 2c). This observation implies that Psb28 is usually released from the PSII intermediate after attachment of CP43, probably after incorporation of PsbJ, as this trigger is missing in the analyzed $\Delta p s b J$ mutant. PSII-I, which only accumulates in the $\Delta p s b J$ mutant, seems to represent a hybrid state of both PSII intermediates that might be stabilized by the incomplete binding of CP43. Psb34 shows sequence similarity to high-light inducible proteins (HLIPs), which play a role in transient chlorophyll storage and chlorophyll biosynthesis $^{53}$. However, the chlorophyll binding motive is missing in Psb34 (Table S2), suggesting a distinct function for this protein in PSII biogenesis.

\section{Psb28 induces conformational changes to the D1 D-E loop}

142 Psb28 binds on the cytosolic faces of the D1 and D2 subunits, directly above the $\mathrm{Q}_{\mathrm{B}}$ binding 143 site (Fig. 3a), which differs from the position that was previously predicted by mass spectrometry ${ }^{45}$. Its binding induces the formation of an extended beta-hairpin structure that incorporates the central anti-parallel beta-sheet of Psb28, the C-terminus of CP47 and the D1 
to the assembly process. In the Psb28-free complex (PSII-M), the CP47 C-terminus blocks the

148 Psb28 binding site by interacting with the D1 D-E loop, thus preventing the reverse process and perturbation of active PSII by Psb28. Using nuclear magnetic resonance (NMR) spectroscopy, we performed chemical shift perturbation (CSP) experiments with recombinant Psb28 and a synthetic peptide of the conserved CP47 C-terminus to characterize this interaction in detail and determine the dissociation constant $\left(\mathrm{K}_{\mathrm{D}}\right)$ (Fig. 3 and Fig. S4). The CSP measurements indicated significant shifts with a chemical shift difference $(\Delta \delta)$ of more than one standard deviation located at strands $\beta 3$ and $\beta 4$ as well at the C-terminal region of $\mathrm{Psb} 28$ (Fig. 3c and d). Upon peptide binding, resonances for several residues gradually appeared with increasing peptide concentration, which were line-broadened beyond detection for the free form of Psb28. This observation indicates a less dynamic and more rigid complex structure. This is further supported by the heteronuclear Overhauser effect (NOE) data, which show that the C-terminus of Psb28 becomes rigid from L108 to K112 upon CP47 peptide binding due to creation of an intermolecular $\beta$-sheet (Fig. 3e). 2D-lineshape analysis was performed, yielding a $K_{D}$ of $53.92 \pm 0.41 \mu \mathrm{M}$ and a dissociation rate $\mathrm{k}_{\text {off }}$ of $10.14 \pm 0.16 \mathrm{~s}^{-1}$, which is consistent with the observed slow-exchange in the NMR spectra (Fig. 3b). The affinity of Psb28 for full-length CP47 and PSII might indeed be even higher due to additional contacts between Psb28 and the D-E loop of D1 (Fig 3a).

\section{Psb28 prevents full association of $\mathrm{CP} 43$ and distorts the $Q_{\mathrm{B}}$-site}

166

167

Binding of Psb28 - with support of Psb34 — causes major structural perturbations at the PSII acceptor side (Supplementary Movies 1 and 2), which mainly involve the D-E loops of the central D1 and D2 subunits. Comparison of the CP43 structure in PSII-I with that in our PsbJ-free control PSII-M (Fig. 4a-d) or with that in mature monomeric PSII (PDB-ID 3KZI) (Fig. 4c and d) reveals several differences. The CP43 C-terminus is not resolved in PSII-I, probably due to an immature position of the last transmembrane helix of CP43 and an altered conformation of the D1 D-E loop, which may prevent binding of the CP43 C-terminus to the cytoplasmic PSII surface (Fig. 4b). This region is close to the loop between helices D and E of the D2 subunit, which is also altered by binding of Psb28, as clearly shown by movement of D2 Arg233 (Fig. 4b, Fig. S5a and b). After dissociation of Psb28, the CP43 module undergoes a rigid body rotation where it clicks into place (Fig. 4b-d, Supplementary Movie 1), whereas binding of PsbJ and the extrinsic proteins PsbO, PsbV and PsbU during further maturation has very little influence on the CP43 binding position (Fig. $4 \mathrm{c}$ and d). The part of PSII that 
originates from RC47 shows almost no difference between PSII-I and mature PSII (Fig. 4d), except for PsbE, which binds adjacent to PsbJ (Fig. 4c).

Most importantly, the structural changes in the D1 D-E loop may have a direct functional impact on PSII electron transfer (Fig. 4e and f), as this region coordinates several important PSII cofactors. In functional PSII, after charge separation at the reaction center $\mathrm{P}_{680}$, electrons are transferred via pheophytin to the bound plastoquinone $\left(\mathrm{Q}_{\mathrm{A}}\right)$ and further to mobile plastoquinone $\left(Q_{B}\right)$. In our PSII-I structure, the $Q_{A}$ site is fully assembled, and a well-resolved $Q_{A}$ molecule is bound (Fig. 4e and f, Fig. S5c and d). The nearby non-heme iron is also already in place in

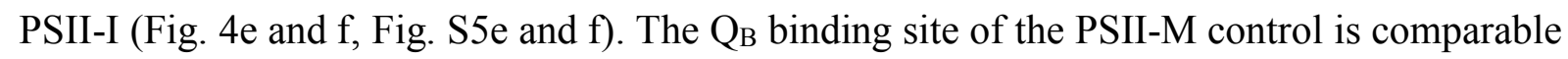
to mature PSII, although it is not occupied by $\mathrm{Q}_{в}$ in our preparation (Fig. S5g). In contrast, the Qв binding site of PSII-I is immature due to the Psb28- and Psb34-induced structural changes in the D1 D-E loop (Fig. 4e and f, Fig. S5h). Notably, D1 Phe265, which coordinates the head group of $\mathrm{Q}_{\mathrm{B}}$ in mature PSII, is clearly at a different position ${ }^{21}$ (Supplementary Movie 2).

\section{Binding of Psb28 protects PSII during biogenesis}

A more detailed analysis of the structural environment close to the $\mathrm{Q}_{\mathrm{A}} / \mathrm{Q}_{\mathrm{B}}$ binding sites revealed differences in the coordination and the hydrogen-bond network of the adjacent non-heme iron, which also indicate functional consequences for PSII electron transfer and charge recombination processes. In mature PSII, the non-heme iron is coordinated by four histidine residues and bicarbonate as the fifth ligand (Fig. 5a and c), whereas in PSII-I, the bicarbonate molecule is replaced by the E241 side-chain of D2 (Fig. 5b and d, Fig. S5e and f, Supplementary Movie 3). Other residues, including D1 E244 and Y246, which bind to the bicarbonate molecule in mature PSII (Fig. 5a), are also displaced in PSII-I due to the conformational change of the D1 D-E loop (Fig. 5b, Fig. S5e and f, Supplementary Movie 3). Binding of bicarbonate is important for PSII efficiency ${ }^{55}$, as it lowers the redox potential of $\left(\mathrm{Q}_{\mathrm{A}} / \mathrm{Q}_{\mathrm{A}}{ }^{-}\right)$to favor forward electron transport ${ }^{56,57}$. If charge recombination occurs, the lower redox potential favors indirect charge recombination via $\mathrm{P}_{680^{\circ}} / \mathrm{Pheo}^{\circ}$. This back reaction yields triplet chlorophyll and subsequently singlet oxygen ${ }^{57}$, a highly reactive oxidizing species. Changes in the redox potential of $\left(\mathrm{Q}_{A} / \mathrm{Q}_{A}{ }^{-}\right)$have been proposed to tune the efficiency of PSII depending on the availability of $\mathrm{CO}_{2}$ as the final electron acceptor and thereby protect PSII under low $\mathrm{CO}_{2}$ conditions ${ }^{57}$. Therefore, we used flash-induced variable fluorescence to monitor the stability of $\mathrm{Q}^{-}{ }^{-}$in active PSII, in the PSII-I assembly intermediate and in inactivated, Mn-depleted PSII, both of which lack a functional OEC (Fig. 5e, Fig. S6a and b). The results indicate that the 
unique fluorescence traces observed with PSII-I are a consequence of $\mathrm{Q}_{\mathrm{A}} / \mathrm{Q}_{\mathrm{A}}{ }^{-}$redox tuning by an absent $\mathrm{OEC}$, an immature $\mathrm{Q}_{\mathrm{B}}$-binding pocket and probably the replacement of bicarbonate

213 by glutamate as a ligand of the none-heme iron (see supplementary discussion for details). To 214 determine the functional consequences of this redox tuning in PSII-I, we measured the formation of ${ }^{1} \mathrm{O}_{2}$ by EPR spectroscopy using the spin probe TEMPD. The data clearly show that ${ }^{1} \mathrm{O}_{2}$ formation is reduced by $\sim 30 \%$ in PSII-I compared to inactivated PSII (Fig. 5f).

\section{Psb27 binds to loop E of CP43 at the luminal PSII surface}

Psb27 binds to the luminal side of the PSII complex, adjacent to loop E of the CP43 subunit (Fig. 6a and b). We could successfully model the Psb27 N-terminus until Cys22, which is the first residue after N-terminal processing of Psb2734. Psb27-Cys22 is located close to the membrane plane, but the lipid moiety is not well enough resolved to build a model. In contrast to previously proposed models ${ }^{58,59}$, the binding site of Psb27 has little overlap with the binding sites of the extrinsic subunits (PsbO, PsbV and PsbU) and has at least no direct contact to the $\mathrm{Mn}_{4} \mathrm{CaO}_{5}$ cluster binding site, particularly not to the D1 C-terminus (Fig. 6a and b), which has been suggested previously ${ }^{58,60}$. Instead, Psb27 is bound at a remote position that might be occupied by CyanoQ in the mature complex ${ }^{61}$. This position of Psb27 may still prevent proper binding of $\mathrm{PsbO}^{34}$ by lowering its binding affinity due to the partial overlap of the binding sites, although both proteins can bind together, as PsbO-containing Psb27-PSII complexes have been isolated via his-tagged Psb2 $7^{49}$. Additionally, Psb27 might stabilize loop E of CP43 in the unassembled state and facilitate its binding to the D1 subunit. This is of particular importance, as loop E of CP43 provides Arg345 and Glu342 (Arg357 and Glu354 in previous publications, see figure caption), to position the $\mathrm{Mn}_{4} \mathrm{CaO}_{5}$ cluster in mature PSII (Fig. 6b, dashed box). Glu342 serves a ligand, whereas $\mathrm{Arg} 345$ stabilizes the $\mathrm{Mn}_{4} \mathrm{CaO}_{5}$ cluster by coordinating Asp170 of D1 through a hydrogen bond. Moreover, in the Psb27-bound state (PSII-I), the D1 C-terminus, which is directly involved in coordination of the $\mathrm{Mn}_{4} \mathrm{CaO}_{5}$ cluster $^{21}$, is bound away from the cluster (Fig. 6c, Fig. S7), which might be an additional consequence of Psb27-induced stabilization of the CP43 E-loop. Thus, our PSII-I structure reveals not only how the Psb27 protein binds to $\mathrm{CP} 43$ and thus stabilizes it, but also indicates a role for the maturation of the oxygen evolving cluster that is consistent with functional data from previous studies ${ }^{32,35,48}$. 
242 The unique $\mathrm{Mn}_{4} \mathrm{CaO}_{5}$ cluster is a key feature of PSII that splits water into oxygen and protons.

243 However, our PSII-I complex does not show any oxygen-evolving activity, suggesting that the 244 oxygen evolving complex (OEC) is not fully assembled. In mature PSII, the $\mathrm{Mn}_{4} \mathrm{CaO}_{5}$ cluster is submerged in the complex and additionally capped by the extrinsic subunits PsbO, PsbU and PsbV (Fig. 6a and b). In our PSII-I structure, these subunits are absent, which leaves two parts of the CP43 E-loop (residues 320-327 and 397-404) in a flexible conformation, exposing the binding site of $\mathrm{Mn}_{4} \mathrm{CaO}_{5}$ cluster to the lumen. There is no strong density feature at this position that would correspond to the fully assembled metal-redox cofactor. Thus, our PSII-I structure provides a model for an immature OEC. Comparing our structure with the high-resolution crystal structure of mature PSII ${ }^{21}$ (PDB-ID 3WU2) provides insights into the first-steps of OEC biogenesis (Fig. 7).

The D1 C-terminus is an essential component for formation of the of OEC, as it provides several charged residues that are responsible for coordination of the chloride ion and the $\mathrm{Mn}_{4} \mathrm{CaO}_{5}$ cluster (Fig. 7a, b and d). The density for these C-terminal residues is weak in our PSII-I map, but traceable (Fig. S7a), indicating a flexibility that confirms the absence of the OEC. Compared to the mature complex, the last 12 residues of the C-terminal tail of D1 would need to undergo significant conformational changes to bring the side chains of Glu333, His337, Asp342, and the Ala344 C-terminus into the correct position to coordinate the $\mathrm{Mn}_{4} \mathrm{CaO}_{5}$ cluster (Supplementary Movie 4).

Moreover, we identify a clearly visible density at the position of the chloride ion, which is coordinated by Lys317 (D2) and the hydrogen atom of the backbone nitrogen of Glu333 (D1) in mature PSII (Fig. 7b and e). Despite the similar position, the $\mathrm{Cl}^{-}$is coordinated by the nitrogen atom of the ring of adjacent His332 (D1) in PSII-I (Fig. 7a and e, Fig. S7d). Surprisingly, we identified another density in the area where the $\mathrm{Mn}_{4} \mathrm{CaO}_{5}$ cluster is located in mature PSII (Fig. 7a-c and f, Fig. S7c). However, this density is not large enough to reflect the whole cluster. Based on its size and interaction partners (Fig. 7f), it corresponds to one positively charged ion. In the structural context, this ion is most likely $\mathrm{Mn}^{2+}$, but it could also be $\mathrm{Ca}^{2+}$ or any other positively charged ion.

270 This ion is coordinated by the side chains of D1 Asp170, Glu189, and His332, which are already 271 in similar positions compared to mature PSII. Glu342 and Arg345 (Glu354 and Arg357 in previous publications) of CP43, which are both involved in the second coordination sphere of the $\mathrm{Mn}_{4} \mathrm{CaO}_{5}$ cluster, are also already pre-positioned through the interaction between $\operatorname{Arg} 345$ 
with D1 Asp170 (Fig. 7g). However, there are still significant conformational changes necessary for the transition from PSII-I to mature PSII, as highlighted in Figure $7 \mathrm{~d}$ and g, as well as in Supplementary Movie 4. The D1 C-terminal tail must bring the side chains of Glu333, His337 and Asp342, as well as the C-terminus of Ala344, into correct alignment to coordinate the $\mathrm{Mn}_{4} \mathrm{CaO}_{5}$ cluster. In addition, the C-terminal tail of D2 needs to flip towards the D1 Cterminus (Fig. 7c, Fig. S7b, Supplementary Movie 4). In summary, PSII-I is characterized by only one positive charged ion bound instead of the complete $\mathrm{Mn}_{4} \mathrm{CaO}_{5}$ cluster, resulting in significantly different conformations of the D1 and D2 C-termini compared to the structural model containing a mature $\mathrm{Mn}_{4} \mathrm{CaO}_{5}$ cluster. However, the PSII-I structure seems to be prepared to accept the $\mathrm{Mn}_{4} \mathrm{CaO}_{5}$ cluster, as indicated by the above described similarities in side chain positioning.

\section{Discussion}

PSII biogenesis is a complex process that requires the action of specific assembly factors. These auxiliary proteins are not present in the mature complex and interact only transiently with specific subunits or preassembled PSII intermediates. Although more than 20 factors have been identified and assigned to specific transitions, their precise molecular functions in PSII assembly remain elusive in almost all cases. Our study provides the first detailed molecular insights into the function of PSII assembly factors Psb27, Psb28 and Psb34, which are involved in an important transition prior to activation of the OEC. The determined binding positions of Psb27 and Psb28, which are two of the most studied PSII assembly factors, define the binding with atomic resolution, in contrast to previous models ${ }^{58,59,62-66}$. This provides precise information about the molecular function of Psb27 and Psb28 in the assembly process.

Binding of Psb28 and Psb34 to the cytoplasmic side of PSII induces large conformational changes in the D1 D-E loop (Fig. 4), which has been identified previously as an important location for PSII photoinhibition and D1 degradation ${ }^{67,68}$. Structural changes observed in the PSII-I Q $\mathrm{Q}_{\mathrm{B}}$ binding pocket and coordination of the non-heme iron suggest a functional impact on PSII electron transfer to protect the immature complex until water splitting is activated. In particular, D2 Glu241 replacing bicarbonate as a ligand of non-heme iron suggests a regulatory role, as binding of bicarbonate was proposed to tune PSII efficiency by changing the redox potential of $\left(\mathrm{Q}_{\mathrm{A}} / \mathrm{Q}_{\mathrm{A}}\right)^{-56,57}$. As a functional consequence, PSII-I generates less singlet oxygen compared to inactivated PSII (Fig. 5f). 
Interestingly, the coordination of the non-heme iron in PSII-I resembles that in non-oxygenic bacterial reaction centers (BRCs) ${ }^{69}$ (Fig. S6c). In BRCs, the fifth ligand of the non-heme iron is provided by E234 of the $\mathrm{M}$ subunit ${ }^{70}$, and mutagenesis of this residue induces changes in the free energy gap between the $\mathrm{P}^{\bullet+} / \mathrm{Q}^{\bullet}{ }^{\bullet-}$ radical pair $^{71}$. These findings indicate that the environment of the non-heme iron is important for the $\mathrm{Q}_{\mathrm{A}}$ redox potential, which influences charge recombination and the formation of reactive oxygen species ${ }^{56}$.

The Psb27-bound and -unbound structures do not differ substantially (Fig. 6b), suggesting a rather subtle action in PSII biogenesis. Previous work demonstrated that Psb27 is already bound to free $\mathrm{CP} 43^{32}$, where it might protect free CP43 from degradation or stabilize the E-loop in a specific conformation to chaperone the subsequent association with the RC47 complex. This step is crucial for preparing the binding site of the $\mathrm{Mn}_{4} \mathrm{CaO}_{5}$ cluster, as the CP43 E-loop is involved in its second coordination sphere ${ }^{72}$. Moreover, a recent high-speed AFM study with spinach PSII has shown fluctuations of the C43 E-loop at room temperature after removal of the extrinsic subunits and destruction of the $\mathrm{Mn}_{4} \mathrm{CaO}_{5}$ cluster $^{73}$, which might suggest a role of Psb27 in restraining the scope of conformational fluctuations for further assembly of the OEC. Consistent with this model, recent spectroscopic analysis of PSII from Synechocystis sp. PCC 6803 revealed that Psb27 facilitates photoactivation, probably by stabilization of intermediates with increased accessibly for ions, especially $\mathrm{Ca}^{2+}$, to the site of cluster assembly ${ }^{74}$. However, our data indicate a self-organization of the CP43 E-loop, at least in T. elongatus. The corresponding structural models for this region are almost identical, independent of binding of Psb27 or the extrinsic proteins, whereas the positions of the D1 and D2 C-termini are clearly different between intermediate and mature PSII. Binding of PsbO seems to be the main driver for these structural differences, and its presence interferes with efficient photoactivation, probably by closing the cluster binding site and thus preventing the exchange of ions ${ }^{74}$. The overlap of the Psb27 and PsbO binding sites, although small, might decrease the PsbO affinity and prevent its premature binding, as initially suggested ${ }^{34,37}$, or Psb27 may promote binding of $\mathrm{PsbO}$ in a different conformation (both proteins can bind simultaneously in a stable complex ${ }^{49}$ ) that might support photoactivation.

Previous structural studies aimed to obtain mechanistic insights into the dark-rearrangement during photoactivation by removing the $\mathrm{Mn}_{4} \mathrm{CaO}_{5}$ cluster from fully assembled PSII, either by depleting it directly from PSII crystals by chemical treatment ${ }^{75}$ or by cryo-EM single particle analysis in manganese- and calcium-free buffer ${ }^{76}$. The X-ray structure was indeed missing the $\mathrm{Mn}_{4} \mathrm{CaO}_{5}$ cluster, but the $\mathrm{D} 1 \mathrm{C}$-terminus followed mostly the same trajectory as found in the mature PSII-dimer structure. The authors suggested that the D1 C-terminus might not rearrange 
during $\mathrm{Mn}_{4} \mathrm{CaO}_{5}$ cluster assembly. However, the crystal structure was dimeric and still had the extrinsic subunits PsbO, PsbU, and PsbV bound. It is known that these subunits are typically not associated with $\mathrm{Mn}_{4} \mathrm{CaO}_{5}$ cluster-depleted PSII. Thus, the structure might be artificially stabilized by crystal packing forces. The cryo-EM structure, on the other hand, revealed a monomeric PSII that lacks extrinsic subunits and the $\mathrm{Mn}_{4} \mathrm{CaO}_{5} \mathrm{cluster}^{76}$. This structure is more similar to our PSII biogenesis intermediate PSII-I, as PsbY, PsbZ and PsbJ are also missing. The PsbJ subunit is surprising; it is an integral subunit of PSII and should not be easily detached, yet it is missing from this structure, as we deleted it to stabilize our PSII-I complex. These observations might indicate a more specific and regulatory role of PsbJ in PSII biogenesis. Additionally, the D1 C-terminus is disordered in this previous cryo-EM structure, and the authors suggest that the dark-rearrangement involves a transition from a disordered to an ordered state.

Our structure now reveals the fate of the D1 C-terminus with the assembly factor Psb27 bound. The D1 C-terminus follows a different trajectory compared to the mature PSII. Thus, we provide structural evidence that the slow dark-rearrangement involves a conformational change of the D1 C-terminus rather than the previously proposed disorder-to-order transition after initial photoactivation ${ }^{76}$. Compared to mature PSII, twelve residues of the D1 C-terminal tail must undergo significant conformational changes to bridge the side chains of Glu333, His337 and Asp342, as well as to bring the C-terminus of Ala344 to the correct position to coordinate the $\mathrm{Mn}_{4} \mathrm{CaO}_{5}$ cluster (Fig. 6c and 7d, Supplementary Movie 4), which is consistent with previous models $39,77,78$. We also identified a single positively charged ion in our PSII-I structure, coordinated by Asp170, Glu189 and His332 of D1 (Fig. 7f), at the position of the $\mathrm{Mn}_{4} \mathrm{CaO}_{5}$ cluster of mature PSII. This binding site most likely corresponds to the long-sought single high-affinity site (HAS), where the first $\mathrm{Mn}^{2+}$ binds prior to the first photoactivation step in OEC biogenesis ${ }^{41}$. The presence of manganese in immature PSII, due to a PratA-facilitated pre-loading mechanism, has been previously suggested ${ }^{79}$. However, we cannot exclude binding of $\mathrm{Ca}^{2+}$, which was shown to bind with a much lower affinity ${ }^{77,80}$, or any other positively charged ion at this position. Nevertheless, Asp170 has been identified as the most critical residue for the HAS ${ }^{81,82}$, which supports our hypothesis. Further photoactivation steps occur presumably after cooperative binding of calcium and manganese. The binding of the extrinsic subunit PsbO, potentially after release of Psb27 and maturation of the water oxidizing complex, is the next step of the PSII assembly line in vivo, which leads to the next unsolved question in PSII biogenesis: what triggers the release of an assembly factor? For Psb27, its detachment might be promoted by the binding of PsbO, as their binding sites partially overlap. 
373 Membrane protein complexes play a fundamental role in bioenergetics to sustain and proliferate

374 life on Earth. They drive the light-to-chemical energy conversion in photosynthetic organisms

375 and are essential for energy supply in heterotrophs. These intricate molecular machines are 376 assembled from numerous single proteins in a spatiotemporally synchronized process that is

377 facilitated by a network of assembly factors. These auxiliary proteins are the key players of

378 Nature's assembly lines. Our PSII-I cryo-EM structure reveals the first molecular snapshot of

379 PSII biogenesis and, accompanied by our spectroscopic and biochemical analyses, provides 380 clear mechanistic insights into how three assembly factors (Psb27, Psb28 and Psb34) coordinate 381 the stepwise construction of this powerful catalyst of life.

\section{Methods}

\section{Cultivation of Thermosynechococcus elongatus BP-1}

384 Cell growth and thylakoid membrane preparation were performed as described previously ${ }^{83}$. In brief, T. elongatus mutant strains ( $\Delta \mathrm{psbJ}$ psbC-TS and psb34-TS) were grown in BG-11 liquid medium inside a 25-litre foil fermenter (Bioengineering) at $45^{\circ} \mathrm{C}, 5 \%(\mathrm{v} / \mathrm{v}) \mathrm{CO}_{2}$-enriched air bubbling and 50-200 $\mu \mathrm{mol}$ photons $\mathrm{m}^{-2} \mathrm{~s}^{-1}$ white light illumination (depending on the cell density). Cells were harvested at an $\mathrm{OD}_{680}$ of $\sim 2$ after 5-6 days of cultivation and concentrated to $\sim 0.5$ 1, using an Amicon DC10 LA hollow fibre system, pelleted (3500 rcf, $45 \mathrm{~min}$ and 25 ${ }^{\circ} \mathrm{C}$ ) and resuspended in $150 \mathrm{ml}$ of Buffer D (100 mM Tris-HCL, pH 7.5, $10 \mathrm{mM} \mathrm{MgCl}_{2}, 10 \mathrm{mM}$ $\mathrm{CaCl}_{2}, 500 \mathrm{mM}$ mannitol and $20 \%$ (w/v) glycerol). The harvested cells were flash-frozen in liquid nitrogen and stored at $-80{ }^{\circ} \mathrm{C}$ until further use.

\section{Preparation of $T$. elongatus mutant strains}

394

Thermosynechococcus elongatus $\Delta \mathrm{psbJ}$ psbC-TS was generated based on the previously described strain $T$. elongatus $\Delta \mathrm{psbJ}^{43}$ that was transformed with the plasmid pCP43-TS. The plasmid is based on pCP34-10His ${ }^{34}$. The His-tag sequence was exchanged with TwinStrep-tag by PCR using the primers CP43TS_rev (5'CCCGATATCTTACTTCTCAAATTGCGGAT GAGACCACGCAGAACCACCAGAACCACCGCCGCTGCCGCCGCCTTTTTCGAACTG CGGGTGGCTCC 3') and NTCP43 (5' TGCTCTAGAATGAAAACTTTGTCTTCCCAGA $\left.3^{\prime}\right)$. The resulting PCR product was ligated back into an empty pCP34-10His backbone using $\mathrm{XbaI}$ and EcoRV restriction endonucleases. T. elongatus BP-1 cells were transformed as described previously ${ }^{84}$. Mutant colonies were selected by frequent re-plating onto agar plates with increasing antibiotic concentrations, stopping at $8 \mu \mathrm{g} / \mathrm{ml}$ of chloramphenicol and $80 \mu \mathrm{g} / \mathrm{ml}$ 
404 of kanamycin. Complete segregation of the mutant was confirmed by PCR with the primers 405 CTCP43DS (5' CCGCTCGAGACCATCCAAGCTTGGCAGCA $3^{\prime}$ ) and NTCP43 (5' TGCTCTAGAATGAAAACTTTGTCTTCCCAGA 3').

407

408

409

410

411

412

413

414

415

416

417

418

419

420

421

422

423

424

425

426

427

428

429

430

431

432

433

434

435

T. elongatus psb34-TS was generated by transformation with the plasmid pPsb34-TS. The plasmid DNA was obtained from TwistBioscience. It consisted of psb34 (ts10063) with a Cterminal TwinStrep-tag and a kanamycin resistance cassette, flanked by ts10063-upstream and downstream regions (900 bp each). T. elongatus BP-1 cells were transformed ${ }^{84}$ and mutant selection took place ${ }^{34}$. Complete segregation of the mutant was verified by PCR. The primers used were ts10063-up-for (5' CATATGGTCTCGCAATTATTTGCCCATGC 3') and ts10063down-rev (5' GGTACCCCG ACACAGTTGATCACCGC 3').

\section{Purification of photosystem II assembly intermediates}

Thawed cells were diluted in $100 \mathrm{ml}$ of Buffer A (100 mM Tris-HCL, pH 7.5, $10 \mathrm{mM} \mathrm{MgCl} 2$ and $10 \mathrm{mM} \mathrm{CaCl}_{2}$ ) and pelleted again $\left(21000 \mathrm{rcf}, 20 \mathrm{~min}\right.$ and $\left.4^{\circ} \mathrm{C}\right)$. The pellet was resuspended in $100 \mathrm{ml}$ of Buffer A with $0.2 \%$ (w/v) lysozyme and dark incubated for $75-90 \mathrm{~min}$ at $37{ }^{\circ} \mathrm{C}$. This was followed by cell disruption by Parr bomb (Parr Instruments Company) and pelleting (21 $000 \mathrm{rcf}, 20 \mathrm{~min}$ and $4^{\circ} \mathrm{C}$ ). All following steps were performed under green illumination. The pellet was resuspended in $150 \mathrm{ml}$ of Buffer A and pelleted again (21 $000 \mathrm{rcf}, 20 \mathrm{~min}$ and $\left.4^{\circ} \mathrm{C}\right)$. This step was repeated three times, with the last resuspension in $80 \mathrm{ml}$ of Buffer $\mathrm{B}(100$ $\mathrm{mM}$ Tris-HCL, pH 7.5, $10 \mathrm{mM} \mathrm{MgCl}, 10 \mathrm{mM} \mathrm{CaCl}_{2}$ and $500 \mathrm{mM}$ mannitol). The isolated thylakoid membranes were flash frozen in liquid nitrogen and stored at $-80{ }^{\circ} \mathrm{C}$.

Strep-Tactin-affinity purification of PsbC-TS and Psb34-TS assembly intermediates were performed under green illumination. Membrane protein extraction was performed as described previously ${ }^{83}$, with certain adaptations. Isolated thylakoid membranes were supplemented with $0.05 \%$ (w/v) n-Dodecyl $\beta$-maltoside (DDM) (Glycon) and pelleted ( $21000 \mathrm{rcf}, 20 \mathrm{~min}$ and $4^{\circ} \mathrm{C}$ ).

The sample was resuspended in extraction buffer (100 mM Tris-HCL, pH 7.5, $10 \mathrm{mM} \mathrm{MgCl}_{2}$, $10 \mathrm{mM} \mathrm{CaCl}_{2}, 1.2 \%(\mathrm{w} / \mathrm{v}) \mathrm{DDM}, 0.5 \%(\mathrm{w} / \mathrm{v})$ sodium-cholate and $0.01 \%(\mathrm{w} / \mathrm{v}) \mathrm{DNase}$ ) to a final chlorophyll concentration of $1 \mathrm{mg} / \mathrm{ml}$ and incubated for $30 \mathrm{~min}$ at $20^{\circ} \mathrm{C}$. The solubilized membrane proteins were ultra-centrifugated (140000 $\mathrm{rcf}, 60 \mathrm{~min}$ and $\left.4{ }^{\circ} \mathrm{C}\right)$ and $\mathrm{NaCl}$ was added to the supernatant to a final concentration of $300 \mathrm{mM}$.

The supernatant was filtered through a $0.45 \mu \mathrm{m}$ syringe filter (Sarsted AG, Germany) and applied to a $5 \mathrm{ml}$ Strep-Tactin Superflow HC column (IBA Lifesciences), equilibrated in Buffer W (100 mM Tris-HCL, pH 7.5, $10 \mathrm{mM} \mathrm{MgCl} 2,10 \mathrm{mM} \mathrm{CaCl}_{2}, 500 \mathrm{mM}$ mannitol, $300 \mathrm{mM}$ 
$\mathrm{NaCl}$ and $0.03 \%(\mathrm{w} / \mathrm{v}) \mathrm{DDM})$ at a flowrate of $3 \mathrm{ml} / \mathrm{min}$. The column was washed with Buffer W until a stable baseline $\left(\mathrm{A}_{280}\right)$ was reached. Strep-tagged protein complexes were eluted by an isocratic elution with Buffer E (100 mM Tris-HCL, pH 7.5, $10 \mathrm{mM} \mathrm{MgCl}_{2}, 10 \mathrm{mM} \mathrm{CaCl}_{2}, 500$ $\mathrm{mM}$ mannitol, $300 \mathrm{mM} \mathrm{NaCl} 2.5 \mathrm{mM}$ desthiobiotin and 0.03\% (w/v) DDM). The captured fractions were equilibrated in Buffer F (20 mM MES, pH 6.5, $10 \mathrm{mM} \mathrm{MgCl}, 10 \mathrm{mM} \mathrm{CaCl}_{2}$, $500 \mathrm{mM}$ mannitol and $0.03 \%(\mathrm{w} / \mathrm{v}) \mathrm{DDM}$ ) with a spin concentrator (Amicon, Ultra - 15, $100000 \mathrm{NMWL})$, flash-frozen in liquid nitrogen and stored at $-80{ }^{\circ} \mathrm{C}$ until analysis.

443 PsbC-TS containing assembly intermediates were further separated by ion exchange chromatography (IEC). Captured elution fraction from the Strep-Tactin-affinity purification were loaded onto a anion exchange column (UNO Q-6, Biorad) with a flowrate of $4 \mathrm{ml} / \mathrm{min}$, pre-equilibrated in Buffer F. Protein complexes were eluted by a liner gradient of $\mathrm{MgSO}_{4}(0-$ $150 \mathrm{mM}$ ) using Buffer G (20 mM MES, pH 6.5, $10 \mathrm{mM} \mathrm{MgCl}, 10 \mathrm{mM} \mathrm{CaCl} 2,500 \mathrm{mM}$ mannitol, $150 \mathrm{mM} \mathrm{MgSO}_{4}$ and $0.03 \%(\mathrm{w} / \mathrm{v})$ DDM). Fractions containing PSII assembly intermediates were collected, concentrated to $100-10 \mu \mathrm{M}$ reaction centers, using a spin concentrator (Amicon, Ultra - 15, 100000 NMWL), aliquoted, flash frozen in liquid nitrogen and stored at $-80{ }^{\circ} \mathrm{C}$ until further analysis.

\section{Cryo-electron microscopy}

453 For cryo-EM sample preparation, $4.5 \mu 1$ of purified protein complexes were applied to glow 454 discharged Quantifoil 2/1 grids, blotted for $3.5 \mathrm{~s}$ with force 4 in a Vitrobot Mark III (Thermo 455 Fisher) at $100 \%$ humidity and $4^{\circ} \mathrm{C}$, then plunge frozen in liquid ethane, cooled by liquid nitrogen. Cryo-EM data was acquired with a FEI Titan Krios transmission electron microscope using the SerialEM software ${ }^{85}$. Movie frames were recorded at a nominal magnification of 22,500x using a K3 direct electron detector (Gatan), The total electron dose of $\sim 55$ electrons per $\AA 2$ was distributed over 30 frames at a calibrated physical pixel size of $1.09 \AA$. Micrographs were recorded in a defocus range of -0.5 to $-3.0 \mu \mathrm{m}$.

\section{Image processing, classification and refinement}

462 Cryo-EM micrographs were processed on the fly using the Focus software package ${ }^{86}$ if they passed the selection criteria (iciness $<1.05$, drift $0.4 \AA<\mathrm{x}<70 \AA$, defocus $0.5 \mathrm{um}<\mathrm{x}<5.5$ um, estimated CTF resolution $<6 \AA$ ). Micrograph frames were aligned using MotionCor $2^{87}$ and the contrast transfer function (CTF) for aligned frames was determined using $\mathrm{Gctf}^{88}$. Using Gautomatch (http://www.mrc-lmb.cam.ac.uk/kzhang/) 693,297 particles were picked templatefree on 824 acquired micrographs. Particles were extracted with a pixel box size of 260 using 
675,123 particles were used for ab initio construction of initial models and subjected to multiple rounds of 3D classification to obtain models with and without Psb28 density. Non-uniform refinement in Cryosparc resulted in models with an estimated resolution of $\sim 3.2 \AA$. Particles belonging to 3D classes with and without Psb28 (150,090 and 166,411 particles, respectively) were reextracted in RELION with a pixel box size of 256 and subjected to several rounds of CTF-refinement (estimation of anisotropic magnification, fit of per-micrograph defocus and astigmatism and beam tilt estimation) and Bayesian polishing ${ }^{91}$. Both classes were refined using the previously generated starting models. 3D classification without further alignment using a mask around the Psb27 region separated particles in the Psb28-containing class into distinct classes with and without Psb27 (57,862 and 91,473 particles, respectively). Final refinement of each of the three classes (with Psb27 and Psb28 (PSII-I), with Psb28 but without Psb27 (PSIII'), and without Psb27 and Psb28 (PSII-M)) resulted in models with global resolutions of 2.94 $\AA, 2.76 \AA$ and $2.82 \AA$, respectively (Gold standard FSC analysis of two independent half-sets at the 0.143 cutoff). Local-resolution and 3D-FSC plots (Extended Data Fig. 2) were calculated using RELION and the "Remote 3DFSC Processing Server" web interface ${ }^{92}$, respectively.

\section{Atomic model construction}

The 3.6 Å resolution X-ray structure of monomeric PSII from T. elongatus with PDB-ID 3KZI ${ }^{52}$ was used as initial structural model that was docked as rigid body using Chimera ${ }^{93}$ into the obtained cryo EM densities for PSII-M and PSII-I. The cofactors that had no corresponding density were removed. The subunit PsbJ was also removed, as it was deleted in the experimental design. By highlighting the still unoccupied parts of the PSII-I density map, we identified densities that lead to the structures of Psb27, Psb28, and Psb34.

The 2.4 Å resolution X-ray structures of isolated Psb28 from T. elongatus with PDB-ID 3ZPN ${ }^{94}$ and the $1.6 \AA$ resolution X-ray structure of isolated Psb27 from T. elongatus with PDB-ID $2 \mathrm{Y} 6 \mathrm{X}^{65}$ were docked as rigid bodies into the unoccupied densities. The 1.6 A resolution X-ray structure of CyanoQ from T. elongatus with PDB-ID 3ZSU ${ }^{95}$ does not fit into the density and was therefore not modeled.

As there was no experimentally resolved structural model of Psb34 available, we first used the sequence with UniProt-ID Q8DMP8 to predict structures using the webserver SWISS Model ${ }^{96}$ and LOMETS $^{97}$. We also predicted the secondary structure through the meta server Bioinformatics Toolkit ${ }^{98}$ and $\mathrm{CCTOP}^{99}$. The results of the secondary structure prediction are summarized in Table S4. Combining these predictions together with the unassigned cryo-EM 
501 density, we used COOT ${ }^{100}$ to build an initial model of Psb34 that has one $\alpha$-helix from amino

502

503

504

505

506

507

508

509

510

511

512

513

514

515

516

517

518

519

520

521

522

523

524

525

526

527

528

529

530

531

532

533 acid number 28 to 55 .

\section{Model Refinement}

The initial model of the complex described above was refined in real space against the cryoEM density of PSII-I, and structural clashes were removed using molecular dynamics flexible fitting (MDFF) ${ }^{101}$. MDFF simulations were prepared in VMD 1.9.4a35 ${ }^{102}$ using QwikMD ${ }^{103}$ and the MDFF plugin. The simulations were carried out with NAMD $2.13^{104}$ employing the CHARMM36 force field. Secondary structure, cis peptide and chirality restraints where employed during 800 steps of minimization followed by a 40 ps MDFF simulation at $300 \mathrm{~K}$. Due to the employed restraints, only conformational changes of side chains and subunit movements compared to the initial structure are identified during the initial MDFF run. We checked the fit to density of the structure by calculating cross-correlation values of the backbone atoms. For PSII-I, we identified residues 217 to 269 from PsbA and residues 467 to 499 from PsbB and PsbZ as main regions where the structural model was not yet in accordance with the density after the initial MDFF run. For these three regions, we employed an iterative combination of MDFF with Rosetta ${ }^{105,106}$. Here, we used the optimized strategy as described for model construction of the $26 \mathrm{~S}$ proteasome ${ }^{107,108}$.

To obtain an atomic model that fit the PSII-M density, we used the initial model based on 3KZI described above, but without PsbJ, Psb27, Psb28, and Psb34. After the initial MDFF run, the cross-correlation check did not reveal any regions with significant deviation between model and density. Therefore, no further refinement was necessary. This fast convergence reflects that there are no crucial differences between the PSII-M model and the X-ray structure 3KZI.

To obtain the atomic model that fit the PSII-I' density, we used the final PSII-I model without Psb27 for MDFF. After the initial MDFF run, the cross-correlation check did not reveal any regions with significant deviation between model and density. This fast convergence reflects that there are no crucial differences between the PSII-I and PSII-I' models, except for the presence of the Psb27 subunit.

Last, the PSII-M, PSII-I, and PSII-I' models were used to initiate one final round of real-space refinement in Phenix ${ }^{109}$.

\section{Acknowledgements}

We thank C. König, M. Völkel, and R. Oworah-Nkruma for excellent technical assistance, Kristin Becker for cloning of the pIVEXPsb28His plasmid, Bibi Erjavec for preparation of the 
534

535

536

537

538

539

540

541

542

543

544

545

546

547

548

549

550

551

552

553

554

555

556

557

558

559

560

561

562

563

564

565

566

567

568

scheme in Fig. 1 and Nicholas Cox for helpful discussion. J.M.S. is grateful to E. Conti for scientific independence and great mentorship and to J. M. Plitzko and W. Baumeister for access to the cryo-EM infrastructure and early career support. M.M.N. is grateful to his mentor M. Rögner for generous support. Funding: Financial support was provided by the Max Planck Society, the Helmholtz Zentrum München, the DFG research unit FOR2092 (EN 1194/1-1 to B.D.E., NO 836/3-2 to M.M.N.), the DFG priority program 2002 (NO 836/4-1 to M.M.N.), the grant NIH P41-GM104601 (to E.T.) and an Emmy-Noether fellowship (SCHU 3364/1-1 to J.M.S). A.K.-L. was supported by the LabEx Saclay Plant Sciences-SPS (grant number ANR10-LABX-0040-SPS) and the French Infrastructure for Integrated Structural Biology (FRISBI; grant number ANR-10-INSB-05). R.S. gratefully acknowledges support from the DFG (INST 213/757-1 FUGG and INST 213/843-1 FUGG). Author contributions: B.D.E., T.R., J.M.S. and M.M.N. conceived the research, prepared the figures, and wrote the manuscript with the contributions of all other authors. M.M.N. coordinated the activities. Preparation of mutants, PSII isolation and biochemical analysis were performed by J.Z., M.M, P.L. and M.M.N. Mass spectrometry analysis was done by J.M.-C. and J.D.L. J.M.S., S.B. and B.D.E. performed the cryo-EM analysis and T.R. built the structural model with the help of S.K.S., A.C. and E.T. Fluorescence spectroscopy was carried out by J.Z. and M.M.N. EPR experiments were conducted by A.K.-L. NMR experiments were conducted and analyzed by O.A. and R.S.. All authors approved the final version of the manuscript. Competing interests: The authors declare no competing interests. Data availability: The cryo-EM density maps will be deposited in the Electron Microscopy Data Bank, the atomic models of the cryo-EM structures in the worldwide Protein Data Bank (wwPDB) and the NMR assignments for Psb28 in the Biological Magnetic Resonance Bank (BMRB), respectively.

\section{References}

1 Hohmann-Marriott, M. F. \& Blankenship, R. E. Evolution of photosynthesis. Annual review of plant biology 62, 515-548, doi:10.1146/annurev-arplant-042110-103811 (2011).

2 Sanchez-Baracaldo, P. \& Cardona, T. On the origin of oxygenic photosynthesis and Cyanobacteria. New Phytol 225, 1440-1446, doi:10.1111/nph.16249 (2020).

3 Vinyard, D. J., Ananyev, G. M. \& Dismukes, G. C. Photosystem II: the reaction center of oxygenic photosynthesis. Annual review of biochemistry 82, 577-606, doi:10.1146/annurev-biochem-070511-100425 (2013).

4 Boekema, E. J. et al. Supramolecular structure of the photosystem II complex from green plants and cyanobacteria. Proc Natl Acad Sci U S A 92, 175-179, doi:10.1073/pnas.92.1.175 (1995). 
Cox, N., Pantazis, D. A. \& Lubitz, W. Current Understanding of the Mechanism of Water Oxidation in Photosystem II and Its Relation to XFEL Data. Annual review of biochemistry 89, 795-820, doi:10.1146/annurev-biochem-011520-104801 (2020).

6 Shen, J. R. The Structure of Photosystem II and the Mechanism of Water Oxidation in Photosynthesis. Annual review of plant biology 66, 23-48, doi:10.1146/annurev-arplant050312-120129 (2015).

Yano, J. et al. Light-dependent production of dioxygen in photosynthesis. Met Ions Life Sci 15, 13-43, doi:10.1007/978-3-319-12415-5 2 (2015).

8 Ferreira, K. N., Iverson, T. M., Maghlaoui, K., Barber, J. \& Iwata, S. Architecture of the photosynthetic oxygen-evolving center. Science 303, 1831-1838, doi:10.1126/science.1093087 (2004).

580

581

582

583

584

585

586

587

588

589

590

591

592

593

594

595

596

597

598

599

600

601

602

603

604

605

606

607

608

609

610

611

612

613

614

615

616

617

618

619

9 Cardona, T., Sedoud, A., Cox, N. \& Rutherford, A. W. Charge separation in photosystem II: a comparative and evolutionary overview. Biochim Biophys Acta 1817, 26-43, doi:10.1016/j.bbabio.2011.07.012 (2012).

10 Holzwarth, A. R. et al. Kinetics and mechanism of electron transfer in intact photosystem II and in the isolated reaction center: pheophytin is the primary electron acceptor. Proc Natl Acad Sci U S A 103, 6895-6900, doi:10.1073/pnas.0505371103 (2006).

11 Müh, F., Glöckner, C., Hellmich, J. \& Zouni, A. Light-induced quinone reduction in photosystem II. Biochim Biophys Acta 1817, 44-65, doi:10.1016/j.bbabio.2011.05.021 (2012).

12 Faller, P. et al. Rapid formation of the stable tyrosyl radical in photosystem II. Proc Natl Acad Sci U S A 98, 14368-14373, doi:10.1073/pnas.251382598 (2001).

13 Roose, J. L., Frankel, L. K., Mummadisetti, M. P. \& Bricker, T. M. The extrinsic proteins of photosystem II: update. Planta 243, 889-908, doi:10.1007/s00425-0152462-6 (2016).

14 Müh, F. \& Zouni, A. Structural basis of light-harvesting in the photosystem II core complex. Protein Sci 29, 1090-1119, doi:10.1002/pro.3841 (2020).

15 Shi, L. X., Hall, M., Funk, C. \& Schröder, W. P. Photosystem II, a growing complex: updates on newly discovered components and low molecular mass proteins. Biochim Biophys Acta 1817, 13-25, doi:10.1016/j.bbabio.2011.08.008 (2012).

16 Stewart, D. H. \& Brudvig, G. W. Cytochrome b559 of photosystem II. Biochim Biophys Acta 1367, 63-87, doi:10.1016/s0005-2728(98)00139-x (1998).

17 Cox, N. et al. Photosynthesis. Electronic structure of the oxygen-evolving complex in photosystem II prior to O-O bond formation. Science 345, 804-808, doi:10.1126/science.1254910 (2014).

18 Kern, J. et al. Structures of the intermediates of Kok's photosynthetic water oxidation clock. Nature 563, 421-425, doi:10.1038/s41586-018-0681-2 (2018).

19 Kupitz, C. et al. Serial time-resolved crystallography of photosystem II using a femtosecond X-ray laser. Nature 513, 261-265, doi:10.1038/nature13453 (2014).

20 Suga, M. et al. An oxyl/oxo mechanism for oxygen-oxygen coupling in PSII revealed by an x-ray free-electron laser. Science 366, 334-338, doi:10.1126/science.aax6998 (2019).

21 Umena, Y., Kawakami, K., Shen, J. R. \& Kamiya, N. Crystal structure of oxygenevolving photosystem II at a resolution of 1.9 A. Nature 473, 55-60, doi:10.1038/nature09913 (2011).

22 Krieger-Liszkay, A., Fufezan, C. \& Trebst, A. Singlet oxygen production in photosystem II and related protection mechanism. Photosynth Res 98, 551-564, doi:10.1007/s11120-008-9349-3 (2008).

23 Vass, I. Molecular mechanisms of photodamage in the Photosystem II complex. Biochim Biophys Acta 1817, 209-217, doi:10.1016/j.bbabio.2011.04.014 (2012). 
24 Shevela, D. et al. 'Birth defects' of photosystem II make it highly susceptible to

621

622

623

624

625

626

627

628

629

630

631

632

633

634

635

636

637

638

639

640

641

642

643

644

645

646

647

648

649

650

651

652

653

654

655

656

657

658

659

660

661

662

663

664

665

666

667

668

669 photodamage during chloroplast biogenesis. Physiologia plantarum 166, 165-180 (2019).

25 Heinz, S., Liauw, P., Nickelsen, J. \& Nowaczyk, M. Analysis of photosystem II biogenesis in cyanobacteria. Biochim Biophys Acta 1857, 274-287, doi:10.1016/j.bbabio.2015.11.007 (2016).

26 Nickelsen, J. \& Rengstl, B. Photosystem II assembly: from cyanobacteria to plants. Annual review of plant biology 64, 609-635, doi:10.1146/annurev-arplant-050312120124 (2013).

27 Nixon, P. J., Michoux, F., Yu, J., Boehm, M. \& Komenda, J. Recent advances in understanding the assembly and repair of photosystem II. Ann Bot 106, 1-16, doi:10.1093/aob/mcq059 (2010).

28 Komenda, J. et al. Cleavage after residue Ala352 in the C-terminal extension is an early step in the maturation of the D1 subunit of Photosystem II in Synechocystis PCC 6803. Biochim Biophys Acta 1767, 829-837, doi:10.1016/j.bbabio.2007.01.005 (2007).

29 Komenda, J. et al. The cyanobacterial homologue of HCF136/YCF48 is a component of an early photosystem II assembly complex and is important for both the efficient assembly and repair of photosystem II in Synechocystis sp. PCC 6803. J Biol Chem 283, 22390-22399, doi:10.1074/jbc.M801917200 (2008).

30 Boehm, M. et al. Subunit composition of CP43-less photosystem II complexes of Synechocystis sp. PCC 6803: implications for the assembly and repair of photosystem II. Philos Trans R Soc Lond B Biol Sci 367, 3444-3454, doi:10.1098/rstb.2012.0066 (2012).

31 Dobáková, M., Sobotka, R., Tichy, M. \& Komenda, J. Psb28 protein is involved in the biogenesis of the photosystem II inner antenna CP47 (PsbB) in the cyanobacterium Synechocystis sp. PCC 6803. Plant Physiol 149, 1076-1086, doi:10.1104/pp.108.130039 (2009).

32 Komenda, J. et al. The Psb27 assembly factor binds to the CP43 complex of photosystem II in the cyanobacterium Synechocystis sp. PCC 6803. Plant Physiol 158, 476-486, doi:10.1104/pp.111.184184 (2012).

33 Mamedov, F., Nowaczyk, M. M., Thapper, A., Rögner, M. \& Styring, S. Functional characterization of monomeric photosystem II core preparations from Thermosynechococcus elongatus with or without the Psb27 protein. Biochemistry 46, 5542-5551, doi:10.1021/bi7000399 (2007).

34 Nowaczyk, M. M. et al. Psb27, a cyanobacterial lipoprotein, is involved in the repair cycle of photosystem II. Plant Cell 18, 3121-3131, doi:10.1105/tpc.106.042671 (2006).

35 Roose, J. L. \& Pakrasi, H. B. The Psb27 protein facilitates manganese cluster assembly in photosystem II. J Biol Chem 283, 4044-4050, doi:10.1074/jbc.M708960200 (2008).

36 Bao, H. \& Burnap, R. L. Photoactivation: The Light-Driven Assembly of the Water Oxidation Complex of Photosystem II. Front Plant Sci 7, 578, doi:10.3389/fpls.2016.00578 (2016).

37 Becker, K., Cormann, K. U. \& Nowaczyk, M. M. Assembly of the water-oxidizing complex in photosystem II. $J$ Photochem Photobiol B 104, 204-211, doi:10.1016/j.jphotobiol.2011.02.005 (2011).

38 Cheniae, G. \& Martin, I. Photoactivation of the manganese catalyst of O2 evolution. IBiochemical and kinetic aspects. Biochim Biophys Acta 253, 167-181, doi:10.1016/0005-2728(71)90242-8 (1971).

39 Dasgupta, J., Ananyev, G. M. \& Dismukes, G. C. Photoassembly of the WaterOxidizing Complex in Photosystem II. Coord Chem Rev 252, 347-360, doi:10.1016/j.ccr.2007.08.022 (2008). 
40 Radmer, R. \& Cheniae, G. M. Photoactivation of the manganese catalyst of O2 evolution. II. A two-quantum mechanism. Biochimica et Biophysica Acta 253, 182-186, doi: 10.1016/0005-2728(71)90243-X (1971).

672

673

41 Nixon, P. J. \& Diner, B. A. Aspartate 170 of the photosystem II reaction center polypeptide D1 is involved in the assembly of the oxygen-evolving manganese cluster. Biochemistry 31, 942-948, doi:10.1021/bi00118a041 (1992).

675

676

Regel, R. E. et al. Deregulation of electron flow within photosystem II in the absence

677 of the PsbJ protein. Journal of Biological Chemistry 276, 41473-41478 (2001).

678 Nowaczyk, M. M. et al. Deletion of psbJ leads to accumulation of Psb27-Psb28 photosystem II complexes in Thermosynechococcus elongatus. Biochim Biophys Acta 1817, 1339-1345, doi:10.1016/j.bbabio.2012.02.017 (2012).

44 Sakata, S., Mizusawa, N., Kubota-Kawai, H., Sakurai, I. \& Wada, H. Psb28 is involved in recovery of photosystem II at high temperature in Synechocystis sp. PCC 6803. Biochim Biophys Acta 1827, 50-59, doi:10.1016/j.bbabio.2012.10.004 (2013).

45 Weisz, D. A. et al. Mass spectrometry-based cross-linking study shows that the Psb28 protein binds to cytochrome b559 in Photosystem II. Proc Natl Acad Sci U S A 114, 2224-2229, doi:10.1073/pnas.1620360114 (2017).

46 Bečková, M. et al. Association of Psb28 and Psb27 Proteins with PSII-PSI Supercomplexes upon Exposure of Synechocystis sp. PCC 6803 to High Light. Mol Plant 10, 62-72, doi:10.1016/j.molp.2016.08.001 (2017).

47 Bentley, F. K., Luo, H., Dilbeck, P., Burnap, R. L. \& Eaton-Rye, J. J. Effects of inactivating psbM and psbT on photodamage and assembly of photosystem II in Synechocystis sp. PCC 6803. Biochemistry 47, 11637-11646, doi:10.1021/bi800804h (2008).

692

693

694

48 Grasse, N. et al. Role of novel dimeric Photosystem II (PSII)-Psb27 protein complex in PSII repair. J Biol Chem 286, 29548-29555, doi:10.1074/jbc.M111.238394 (2011).

49 Liu, H., Roose, J. L., Cameron, J. C. \& Pakrasi, H. B. A genetically tagged Psb27 protein allows purification of two consecutive photosystem II (PSII) assembly intermediates in Synechocystis 6803, a cyanobacterium. J Biol Chem 286, 24865-24871, doi:10.1074/jbc.M111.246231 (2011).

50 Weisz, D. A. et al. A novel chlorophyll protein complex in the repair cycle of photosystem II. Proc Natl Acad Sci $U$ S A 116, 21907-21913, doi:10.1073/pnas.1909644116 (2019).

51 Mabbitt, P. D., Wilbanks, S. M. \& Eaton-Rye, J. J. Structure and function of the hydrophilic Photosystem II assembly proteins: Psb27, Psb28 and Ycf48. Plant Physiol Biochem 81, 96-107, doi:10.1016/j.plaphy.2014.02.013 (2014).

52 Broser, M. et al. Crystal structure of monomeric photosystem II from Thermosynechococcus elongatus at 3.6-Å resolution. J Biol Chem 285, 26255-26262, doi:10.1074/jbc.M110.127589 (2010).

53 Komenda, J. \& Sobotka, R. Cyanobacterial high-light-inducible proteins--Protectors of chlorophyll-protein synthesis and assembly. Biochim Biophys Acta 1857, 288-295, doi:10.1016/j.bbabio.2015.08.011 (2016).

54 Mulo, P. et al. Mutagenesis of the D-E loop of photosystem II reaction centre protein D1. Function and assembly of photosystem II. Plant Mol Biol 33, 1059-1071, doi:10.1023/a:1005765305956 (1997).

55 Eaton-Rye, J. J. \& Govindjee. Electron transfer through the quinone acceptor complex of photosystem II in bicarbonate-depleted spinach thylakoid membranes as a function of actinic flash number and frequency. Biochimica et Biophysica Acta (BBA)Bioenergetics 935, 237-247 (1988).

Allen, J. F. \& Nield, J. Redox Tuning in Photosystem II. Trends Plant Sci 22, 97-99, doi:10.1016/j.tplants.2016.11.011 (2017). 
Brinkert, K., De Causmaecker, S., Krieger-Liszkay, A., Fantuzzi, A. \& Rutherford, A. W. Bicarbonate-induced redox tuning in Photosystem II for regulation and protection. Proc Natl Acad Sci U S A 113, 12144-12149, doi:10.1073/pnas.1608862113 (2016). Cormann, K. U., Möller, M. \& Nowaczyk, M. M. Critical Assessment of Protein CrossLinking and Molecular Docking: An Updated Model for the Interaction Between Photosystem II and Psb27. Front Plant Sci 7, 157, doi:10.3389/fpls.2016.00157 (2016).

59 Liu, H., Huang, R. Y., Chen, J., Gross, M. L. \& Pakrasi, H. B. Psb27, a transiently associated protein, binds to the chlorophyll binding protein CP43 in photosystem II assembly intermediates. Proc Natl Acad Sci $U$ S A 108, 18536-18541, doi:10.1073/pnas.1111597108 (2011).

60 Wei, L. et al. LPA19, a Psb27 homolog in Arabidopsis thaliana, facilitates D1 protein precursor processing during PSII biogenesis. Journal of Biological Chemistry 285, 21391-21398 (2010).

61 Wei, X. et al. Structure of spinach photosystem II-LHCII supercomplex at 3.2 A resolution. Nature 534, 69-74, doi:10.1038/nature18020 (2016).

62 Cormann, K. U. et al. Structure of Psb27 in solution: implications for transient binding to photosystem II during biogenesis and repair. Biochemistry 48, 8768-8770, doi:10.1021/bi9012726 (2009).

63 Fagerlund, R. D. \& Eaton-Rye, J. J. The lipoproteins of cyanobacterial photosystem II. J Photochem Photobiol B 104, 191-203, doi:10.1016/j.jphotobiol.2011.01.022 (2011).

64 Liu, H. et al. Mass spectrometry-based footprinting reveals structural dynamics of loop E of the chlorophyll-binding protein CP43 during photosystem II assembly in the cyanobacterium Synechocystis 6803. J Biol Chem 288, 14212-14220, doi:10.1074/jbc.M113.467613 (2013).

65 Michoux, F. et al. Crystal structure of the Psb27 assembly factor at 1.6 A: implications for binding to Photosystem II. Photosynth Res 110, 169-175, doi:10.1007/s11120-0119712-7 (2012).

66 Weisz, D. A., Gross, M. L. \& Pakrasi, H. B. The Use of Advanced Mass Spectrometry to Dissect the Life-Cycle of Photosystem II. Front Plant Sci 7, 617, doi:10.3389/fpls.2016.00617 (2016).

67 Kettunen, R., Tyystjarvi, E. \& Aro, E. M. Degradation pattern of photosystem II reaction center protein $\mathrm{D} 1$ in intact leaves. The major photoinhibition-induced cleavage site in D1 polypeptide is located amino terminally of the DE loop. Plant Physiol 111, 1183-1190, doi:10.1104/pp.111.4.1183 (1996).

68 Mulo, P., Laakso, S., Maenpaa, P. \& Aro, E. M. Stepwise photoinhibition of photosystem II. Studies with Synechocystis species PCC 6803 mutants with a modified D-E loop of the reaction center polypeptide D1. Plant Physiol 117, 483-490, doi:10.1104/pp.117.2.483 (1998).

69 Stowell, M. H. et al. Light-induced structural changes in photosynthetic reaction center: implications for mechanism of electron-proton transfer. Science 276, 812-816, doi:10.1126/science.276.5313.812 (1997).

70 Wang, X. et al. Is bicarbonate in Photosystem II the equivalent of the glutamate ligand to the iron atom in bacterial reaction centers? Biochim Biophys Acta 1100, 1-8, doi:10.1016/0005-2728(92)90119-m (1992).

71 Cheap, H. et al. M234Glu is a component of the proton sponge in the reaction center from photosynthetic bacteria. Biochim Biophys Acta 1787, 1505-1515, doi:10.1016/j.bbabio.2009.07.004 (2009).

72 Burnap, R. L. D1 protein processing and Mn cluster assembly in light of the emerging Photosystem II structure. Physical Chemistry Chemical Physics 6, 4803-4809 (2004).

73 Tokano, T., Kato, Y., Sugiyama, S., Uchihashi, T. \& Noguchi, T. Structural dynamics of a protein domain relevant to the water-oxidizing complex in photosystem II as 
visualized by high-speed atomic force microscopy. The Journal of Physical Chemistry $B$ 124, 5847-5857 (2020).

74 Avramov, A. P., Hwang, H. J. \& Burnap, R. L. The role of Ca2+ and protein scaffolding in the formation of nature's water oxidizing complex. Proceedings of the National Academy of Sciences 117, 28036-28045 (2020). Zhang, M. et al. Structural insights into the light-driven auto-assembly process of the water-oxidizing Mn4CaO5-cluster in photosystem II. Elife 6, doi:10.7554/eLife.26933 (2017).

76 Gisriel, C. J. et al. Cryo-EM Structure of Monomeric Photosystem II from Synechocystis sp. PCC 6803 Lacking the Water-Oxidation Complex. Joule (2020).

77 Kolling, D. R., Cox, N., Ananyev, G. M., Pace, R. J. \& Dismukes, G. C. What are the oxidation states of manganese required to catalyze photosynthetic water oxidation? Biophys J 103, 313-322, doi:10.1016/j.bpj.2012.05.031 (2012).

78 Zaltsman, L., Ananyev, G. M., Bruntrager, E. \& Dismukes, G. C. Quantitative kinetic model for photoassembly of the photosynthetic water oxidase from its inorganic constituents: requirements for manganese and calcium in the kinetically resolved steps. Biochemistry 36, 8914-8922, doi:10.1021/bi970187f (1997).

79 Stengel, A. et al. Initial steps of photosystem II de novo assembly and preloading with manganese take place in biogenesis centers in Synechocystis. The Plant Cell 24, 660675 (2012).

80 Tyryshkin, A. M. et al. Spectroscopic evidence for $\mathrm{Ca} 2+$ involvement in the assembly of the $\mathrm{Mn} 4 \mathrm{Ca}$ cluster in the photosynthetic water-oxidizing complex. Biochemistry 45, 12876-12889, doi:10.1021/bi061495t (2006).

81 Campbell, K. A. et al. Dual-mode EPR detects the initial intermediate in photoassembly of the photosystem II Mn cluster: the influence of amino acid residue 170 of the D1 polypeptide on Mn coordination. Journal of the American Chemical Society 122, 37543761 (2000).

82 Cohen, R. O., Nixon, P. J. \& Diner, B. A. Participation of the C-terminal region of the D1-polypeptide in the first steps in the assembly of the Mn4Ca cluster of photosystem II. J Biol Chem 282, 7209-7218, doi:10.1074/jbc.M606255200 (2007).

83 Kuhl, H. et al. Towards structural determination of the water-splitting enzyme. Purification, crystallization, and preliminary crystallographic studies of photosystem II from a thermophilic cyanobacterium. $J$ Biol Chem 275, 20652-20659, doi:10.1074/jbc.M001321200 (2000).

84 Iwai, M., Katoh, H., Katayama, M. \& Ikeuchi, M. Improved genetic transformation of the thermophilic cyanobacterium, Thermosynechococcus elongatus BP-1. Plant Cell Physiol 45, 171-175, doi:10.1093/pcp/pch015 (2004).

85 Mastronarde, D. N. Automated electron microscope tomography using robust prediction of specimen movements. Journal of structural biology 152, 36-51 (2005).

86 Biyani, N. et al. Focus: The interface between data collection and data processing in cryo-EM. J Struct Biol 198, 124-133, doi:10.1016/j.jsb.2017.03.007 (2017).

87 Zheng, S. Q. et al. MotionCor2: anisotropic correction of beam-induced motion for improved cryo-electron microscopy. Nat Methods 14, 331-332, doi:10.1038/nmeth.4193 (2017).

88 Zhang, K. Gctf: Real-time CTF determination and correction. J Struct Biol 193, 1-12, doi:10.1016/j.jsb.2015.11.003 (2016).

89 Scheres, S. H. A Bayesian view on cryo-EM structure determination. J Mol Biol 415, 406-418, doi:10.1016/j.jmb.2011.11.010 (2012).

90 Punjani, A., Rubinstein, J. L., Fleet, D. J. \& Brubaker, M. A. cryoSPARC: algorithms for rapid unsupervised cryo-EM structure determination. Nat Methods 14, 290-296, doi:10.1038/nmeth.4169 (2017). 
82391 Scheres, S. H. Beam-induced motion correction for sub-megadalton cryo-EM particles.

$824 \quad$ Elife 3, e03665, doi:10.7554/eLife.03665 (2014).

82592 Tan, Y. Z. et al. Addressing preferred specimen orientation in single-particle cryo-EM through tilting. Nat Methods 14, 793-796, doi:10.1038/nmeth.4347 (2017).

93 Pettersen, E. F. et al. UCSF Chimera--a visualization system for exploratory research and analysis. J Comput Chem 25, 1605-1612, doi:10.1002/jcc.20084 (2004).

94 Bialek, W. et al. Crystal structure of the Psb28 accessory factor of Thermosynechococcus elongatus photosystem II at 2.3 A. Photosynth Res 117, 375383, doi:10.1007/s11120-013-9939-6 (2013).

95 Michoux, F. et al. Crystal structure of CyanoQ from the thermophilic cyanobacterium Thermosynechococcus elongatus and detection in isolated photosystem II complexes. Photosynth Res 122, 57-67, doi:10.1007/s11120-014-0010-z (2014).

96 Schwede, T., Kopp, J., Guex, N. \& Peitsch, M. C. SWISS-MODEL: An automated protein homology-modeling server. Nucleic Acids Res 31, 3381-3385, doi:10.1093/nar/gkg520 (2003).

97 Wu, S. \& Zhang, Y. LOMETS: a local meta-threading-server for protein structure prediction. Nucleic Acids Res 35, 3375-3382, doi:10.1093/nar/gkm251 (2007).

98 Zimmermann, L. et al. A Completely Reimplemented MPI Bioinformatics Toolkit with a New HHpred Server at its Core. $J$ Mol Biol 430, 2237-2243, doi:10.1016/j.jmb.2017.12.007 (2018).

99 Dobson, L., Remenyi, I. \& Tusnady, G. E. CCTOP: a Consensus Constrained TOPology prediction web server. Nucleic Acids Res 43, W408-412, doi:10.1093/nar/gkv451 (2015).

845

846

847

848

849

850

851

852

853

854

855

856

857

858

859

860

861

862

863

864

865

866

867

868

869

870

871

100 Emsley, P., Lohkamp, B., Scott, W. G. \& Cowtan, K. Features and development of Coot. Acta Crystallogr D Biol Crystallogr 66, 486-501, doi:10.1107/S0907444910007493 (2010).

101 Trabuco, L. G., Villa, E., Schreiner, E., Harrison, C. B. \& Schulten, K. Molecular dynamics flexible fitting: a practical guide to combine cryo-electron microscopy and Xray crystallography. Methods 49, 174-180, doi:10.1016/j.ymeth.2009.04.005 (2009).

102 Humphrey, W., Dalke, A. \& Schulten, K. VMD: visual molecular dynamics. J Mol Graph 14, 33-38, doi:10.1016/0263-7855(96)00018-5 (1996).

103 Ribeiro, J. V. et al. QwikMD - Integrative Molecular Dynamics Toolkit for Novices and Experts. Sci Rep 6, 26536, doi:10.1038/srep26536 (2016).

104 Phillips, J. C. et al. Scalable molecular dynamics with NAMD. J Comput Chem 26, 1781-1802, doi:10.1002/jcc.20289 (2005).

105 Leaver-Fay, A. et al. ROSETTA3: an object-oriented software suite for the simulation and design of macromolecules. Methods Enzymol 487, 545-574, doi:10.1016/B978-012-381270-4.00019-6 (2011).

106 Lindert, S. \& McCammon, J. A. Improved cryoEM-Guided Iterative Molecular Dynamics--Rosetta Protein Structure Refinement Protocol for High Precision Protein Structure Prediction. J Chem Theory Comput 11, 1337-1346, doi:10.1021/ct500995d (2015).

107 Guo, Q. et al. In Situ Structure of Neuronal C9orf72 Poly-GA Aggregates Reveals Proteasome Recruitment. Cell 172, 696-705 e612, doi:10.1016/j.cell.2017.12.030 (2018).

108 Wehmer, M. et al. Structural insights into the functional cycle of the ATPase module of the 26S proteasome. Proc Natl Acad Sci $U$ S A 114, 1305-1310, doi:10.1073/pnas.1621129114 (2017).

109 Liebschner, D. et al. Macromolecular structure determination using X-rays, neutrons and electrons: recent developments in Phenix. Acta Crystallogr D Struct Biol 75, 861877, doi:10.1107/S2059798319011471 (2019). 
a

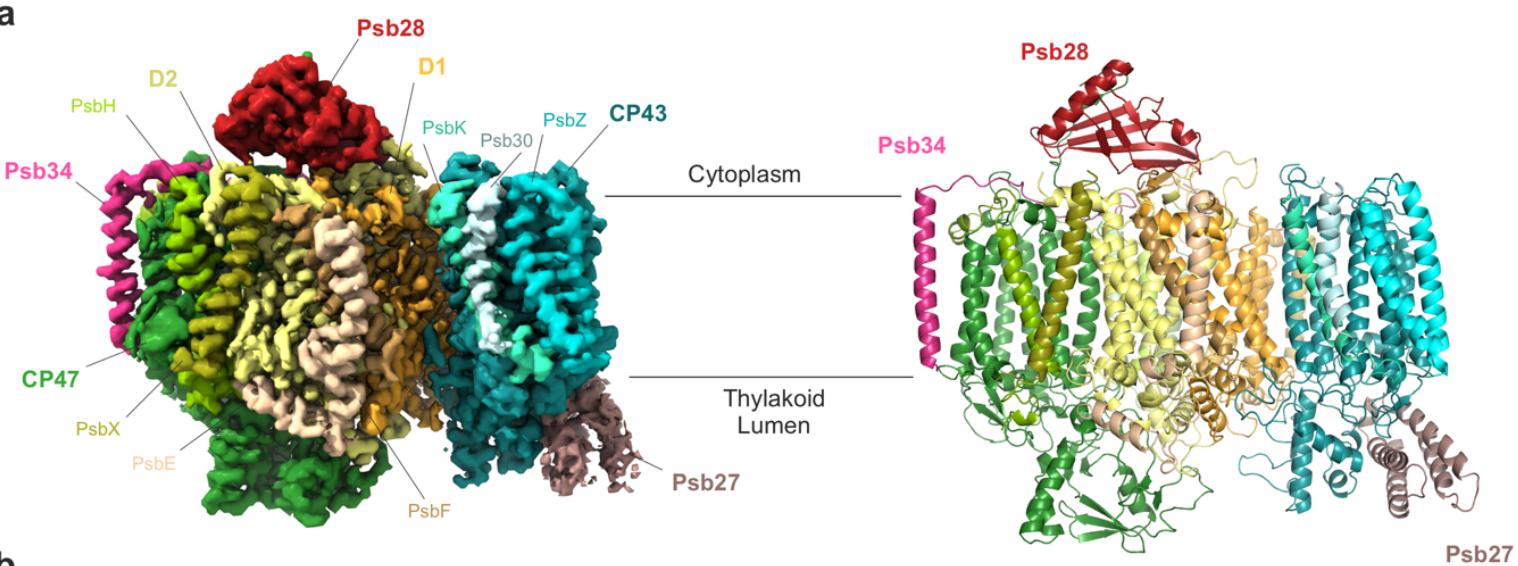

b

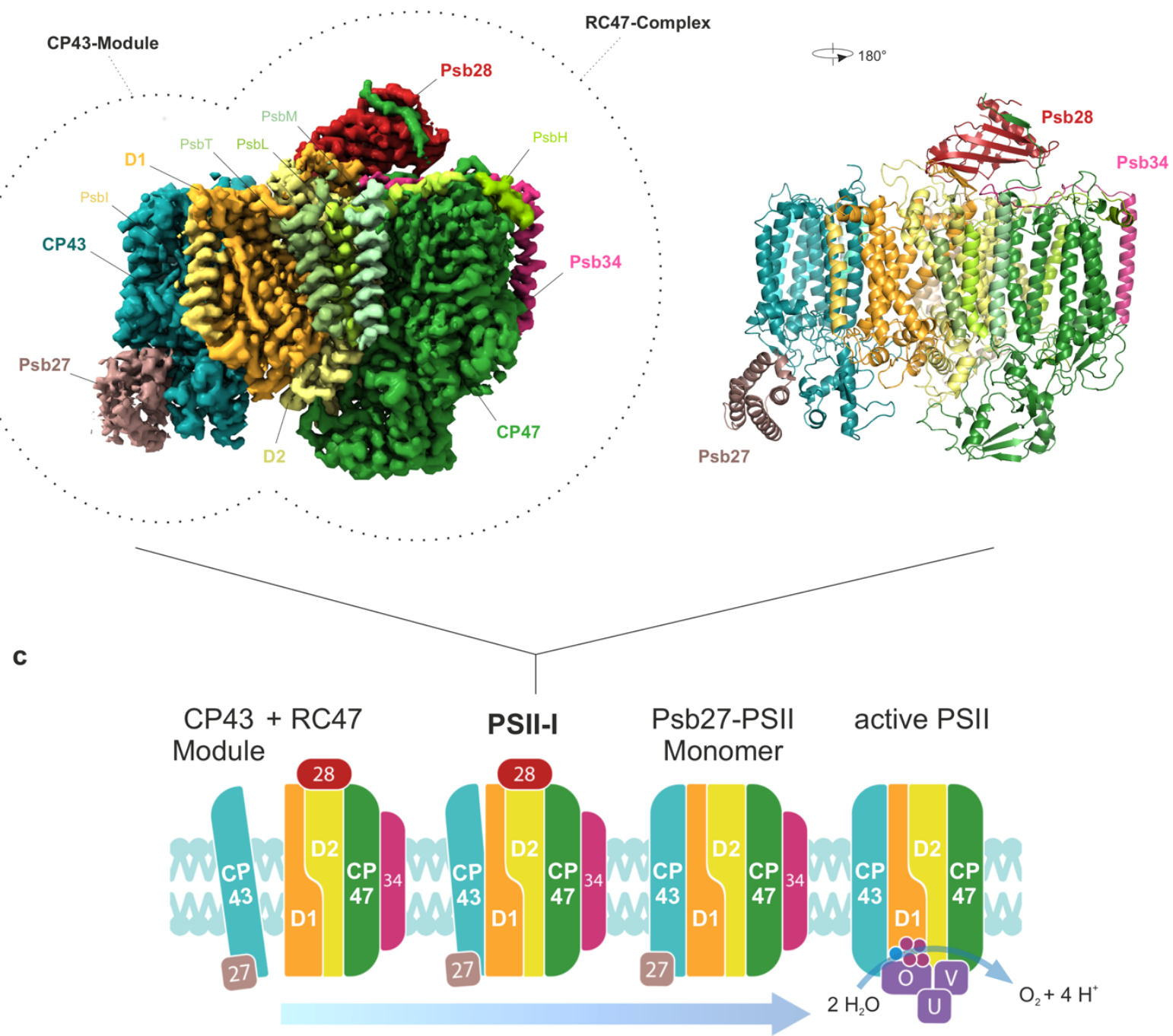

Fig. 1: Cryo-EM map of a PSII assembly intermediate (PSII-I) from T. elongatus, segmented by subunit. a, 15 PSII subunits and 3 assembly factors are colored and named (PSII subunits: D1, D2, CP43, CP47, PsbE, PsbF, PsbH, PsbI, PsbK, PsbL, PsbM, PsbT, PsbX, PsbZ and Psb30; assembly factors: Psb27, Psb28 and ts10063, which we named Psb34) (front view). b, Parts of PSII that originate from the CP43 module (comprised of CP43, Psb27, PsbZ, Psb30 and PsbK) and the RC47 complex are indicated by dashed lines (back view). c, Schematic model of the PSII assembly process starting with the formation of PSII-I from the CP43 module and RC47. Small PSII subunits were omitted for simplicity. 
a
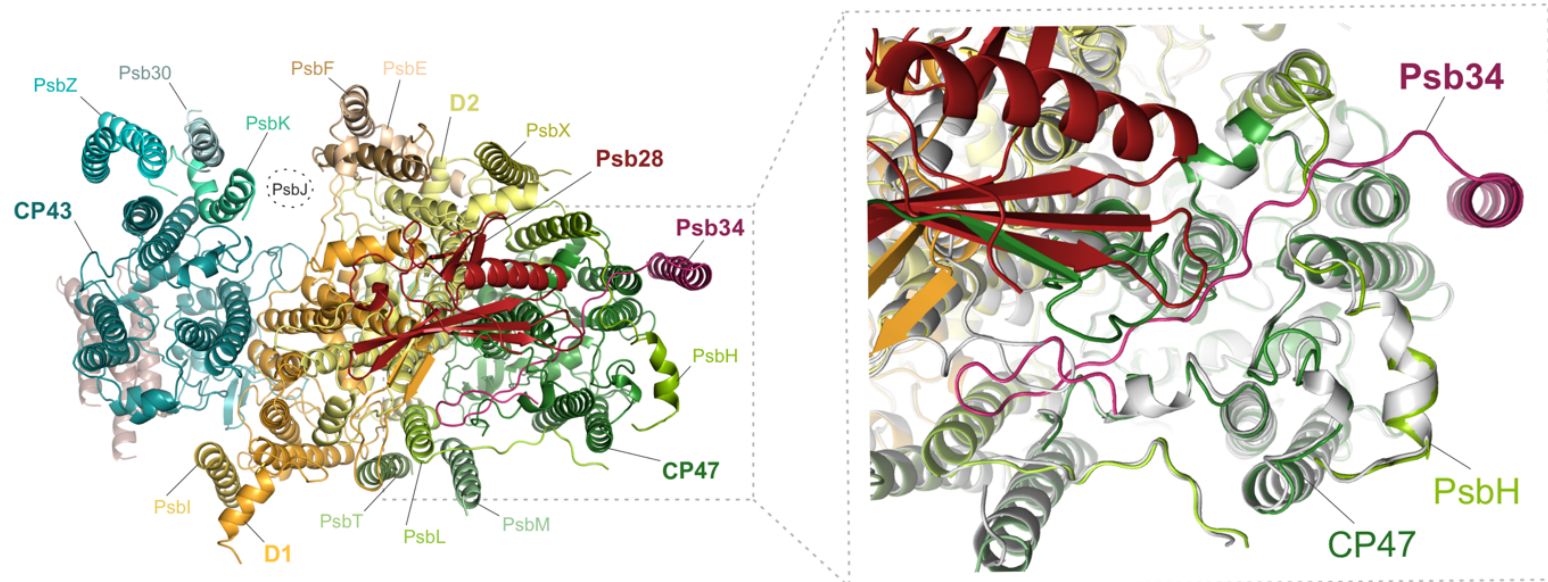

b
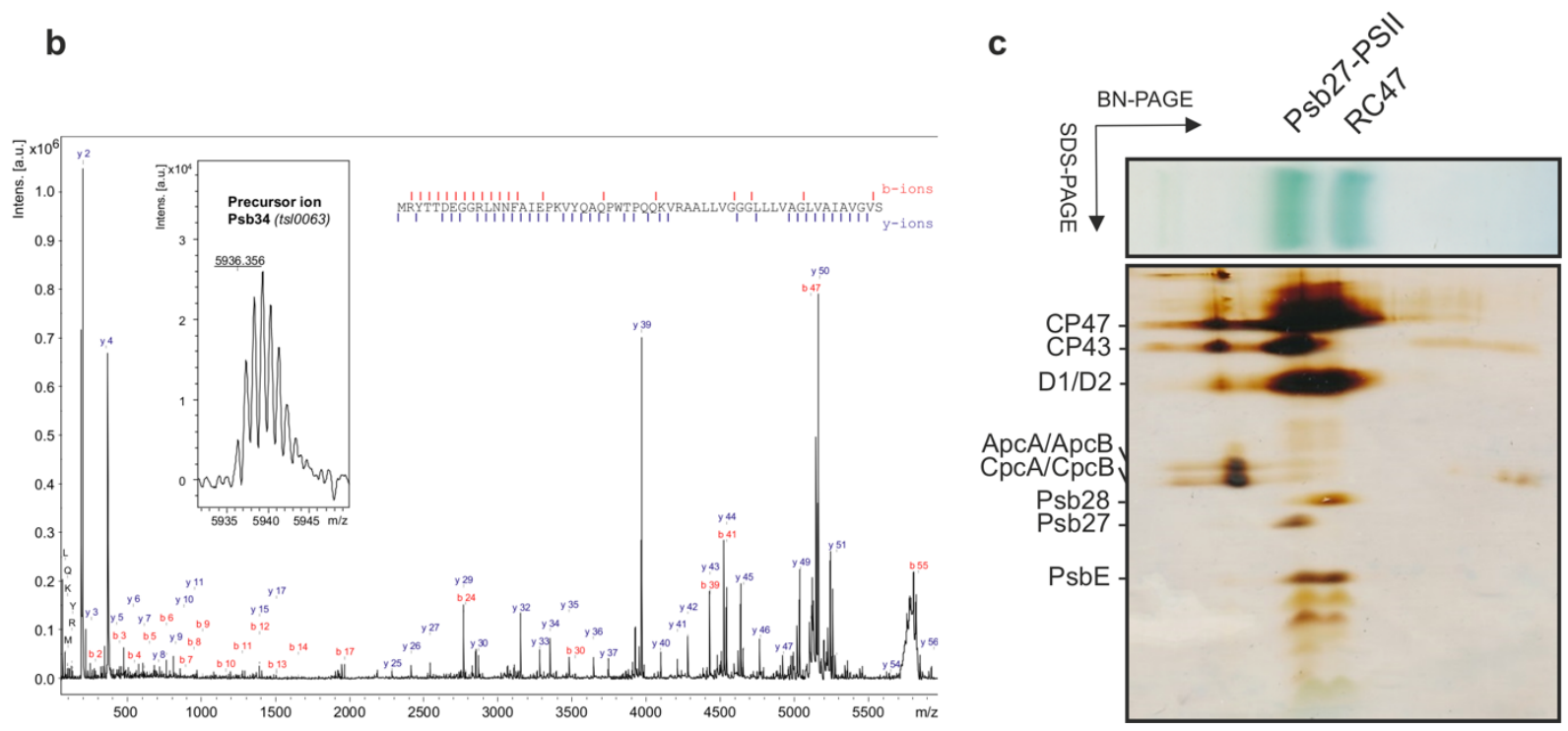

Fig. 2: Psb34 binds to RC47 during attachment of the CP43 module. a, Binding site of Psb34 at CP47, close to PsbH (top view), with extended binding of the Psb34 N-terminus along the cytoplasmic PSII surface (dashed box). b, MALDI-ToF analysis of PSII assembly intermediates. Mass spectrum of Psb34 (ts10063) from the PSII complex (inset) and the fragment spectrum obtained for $\mathrm{m} / \mathrm{z} 5936.356$ with annotated $\mathrm{b}$ - and y-ion series matching the Psb34 sequence. Observed fragmentation sites are indicated by dashes in the sequence. Mascot score: 171. c, Subunit composition of Psb34-PSII assembly intermediates analyzed by 2D-PAGE. 
a

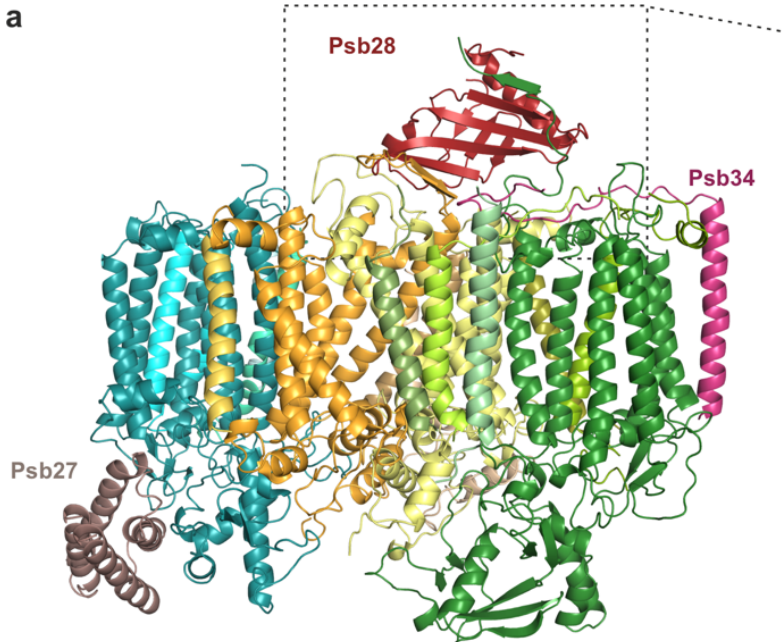

b

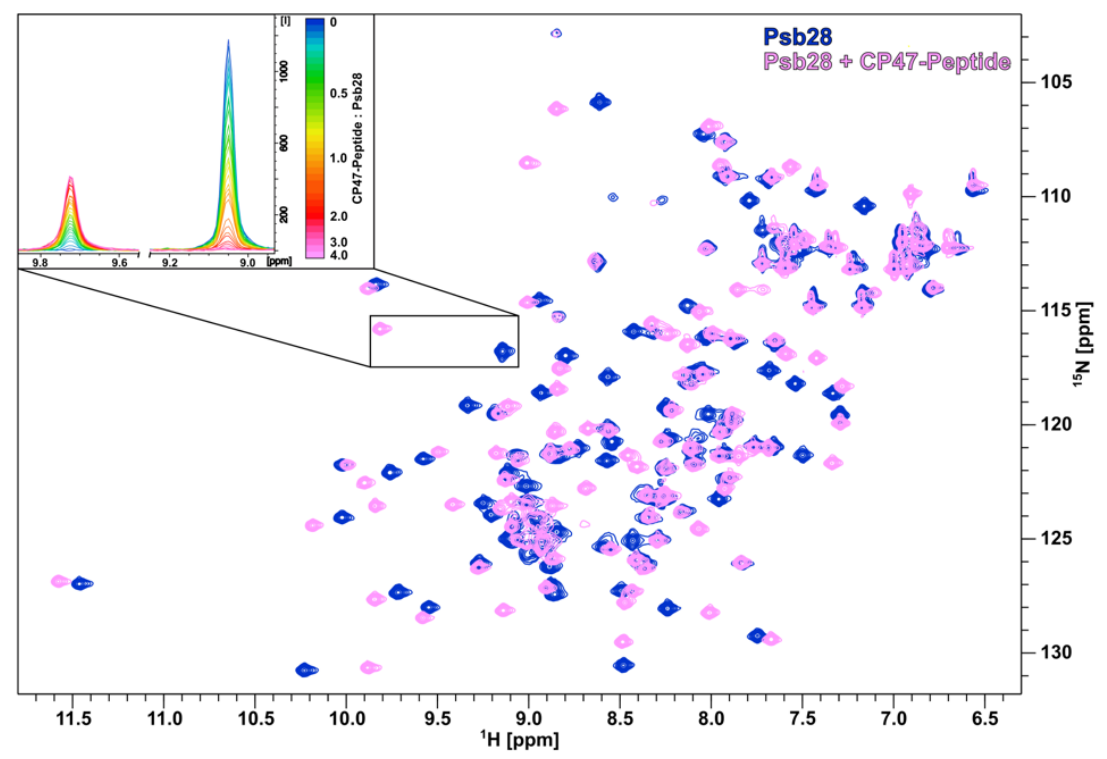

C

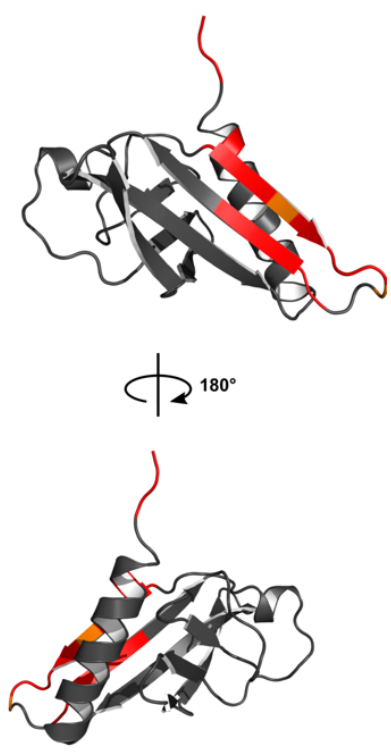

d

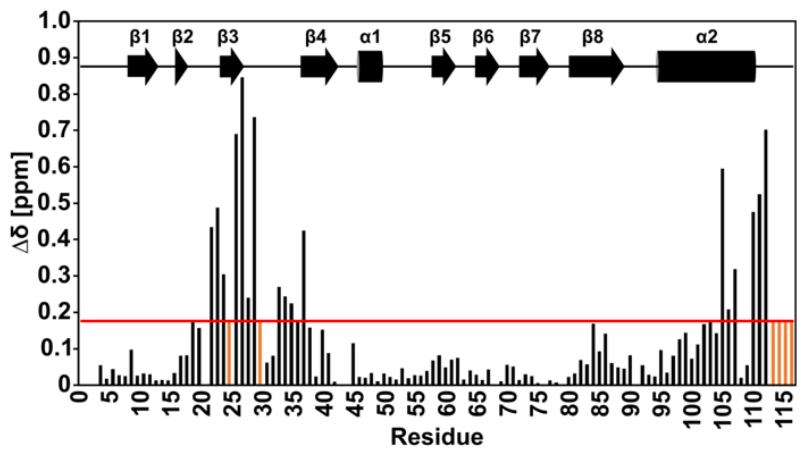

e

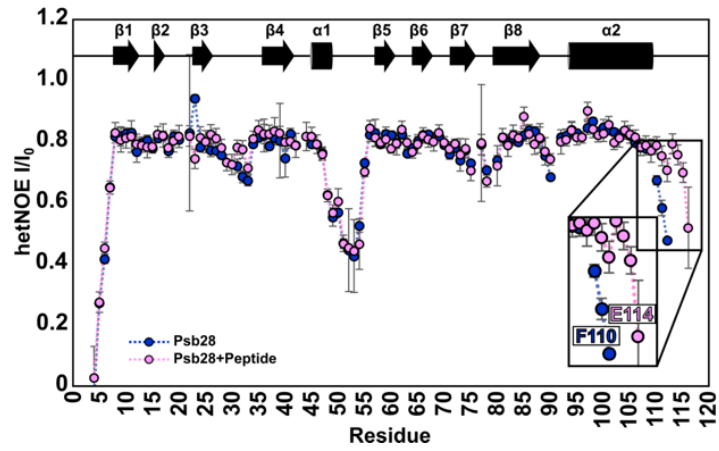

Fig. 3: The role of the CP47 C-terminus in binding of Psb28. a, Binding of Psb28 at the cytoplasmic/stromal PSII surface (side view, colors correspond to Fig. 1) and continuation of the central Psb28 beta-sheet by the CP47 C-terminus and the D-E loop of D1 (dashed box). For comparison, mature monomeric PSII (PDB-ID 3KZI) is shown in gray. b, Superimposed 2D ${ }^{1} \mathrm{H}^{-15} \mathrm{~N}-\mathrm{HSQC}$ spectra of free Psb28 (blue) and Psb28 bound to the C-terminal peptide of CP47 (magenta). Upper left inset: representation of slow exchange behavior for the proton amide resonance of T24, ranging from $126.9 \mathrm{ppm}$ to $128.6 \mathrm{ppm}$ in the ${ }^{15} \mathrm{~N}$ dimension. c, CSPs of more than one SD projected onto the model representation of Psb28. d, Weighted ${ }^{1} \mathrm{H} /{ }^{15} \mathrm{~N}$ chemical shift perturbations observed for 
Psb28 upon binding to the CP47 peptide. Red line indicates one standard deviation (SD), residues that yield resonances only in the complex form are indicated in orange. e, Backbone ${ }^{15} \mathrm{~N}\left\{{ }^{1} \mathrm{H}\right\}$-heteronuclear NOE of free Psb28 (blue) and Psb28 bound to the C-terminal region of the CP47 peptide (magenta). Smaller I/I0 ratios correspond to regions that exhibit dynamics on the pico- to nanosecond timescale. 
a

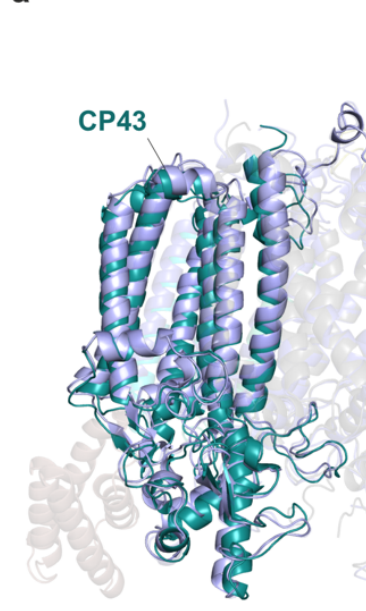

C side view

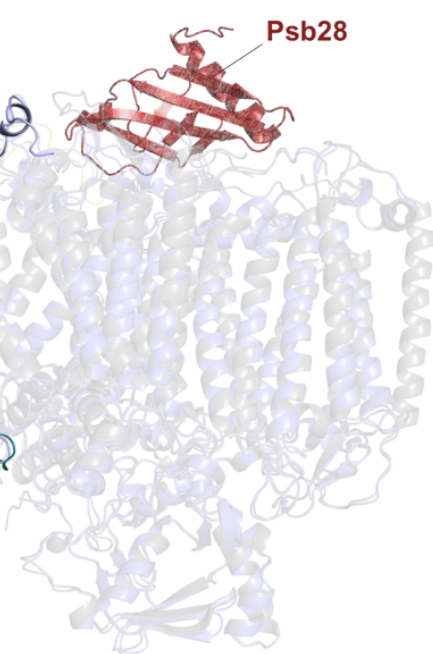

b

top view

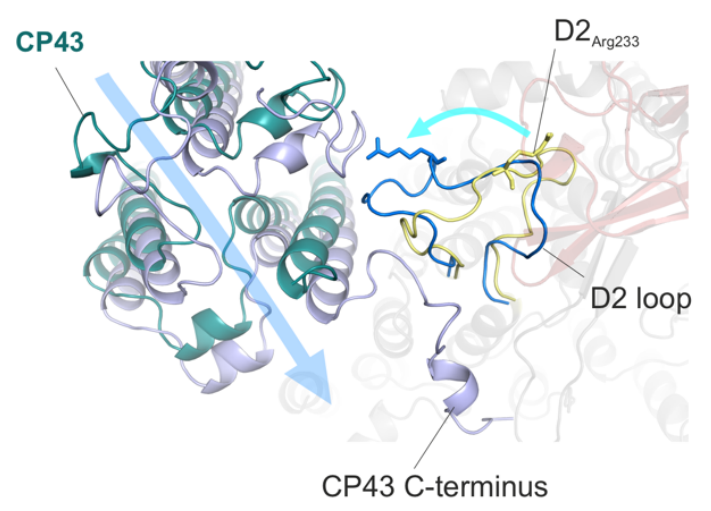

d

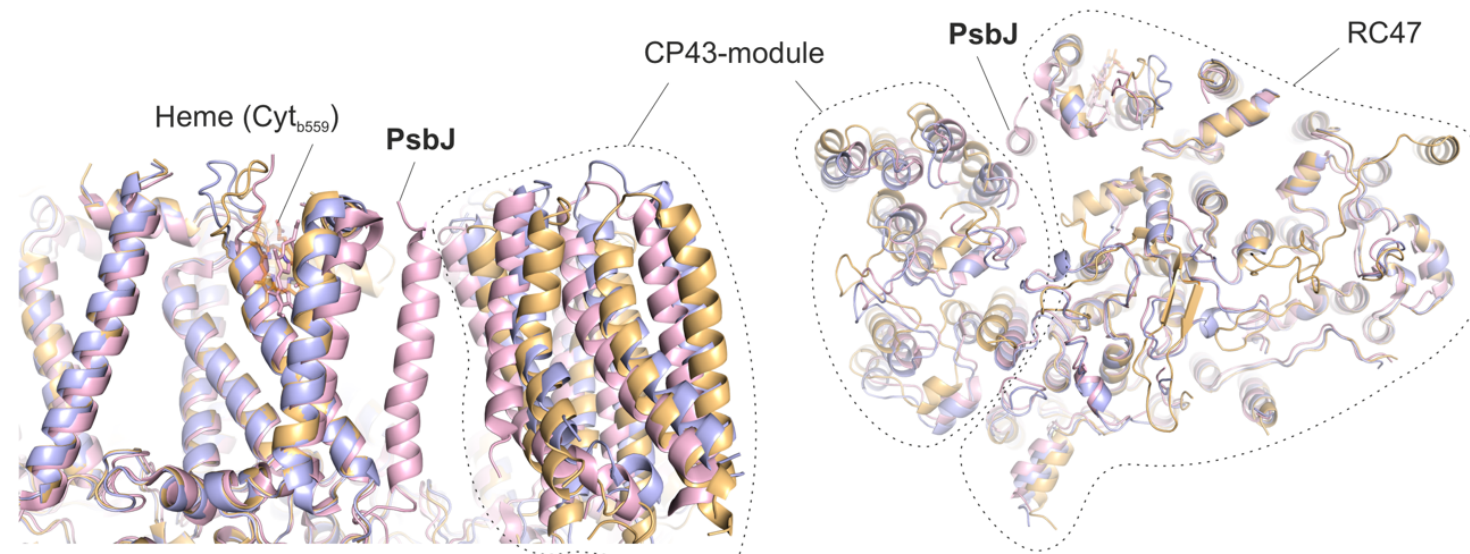

e

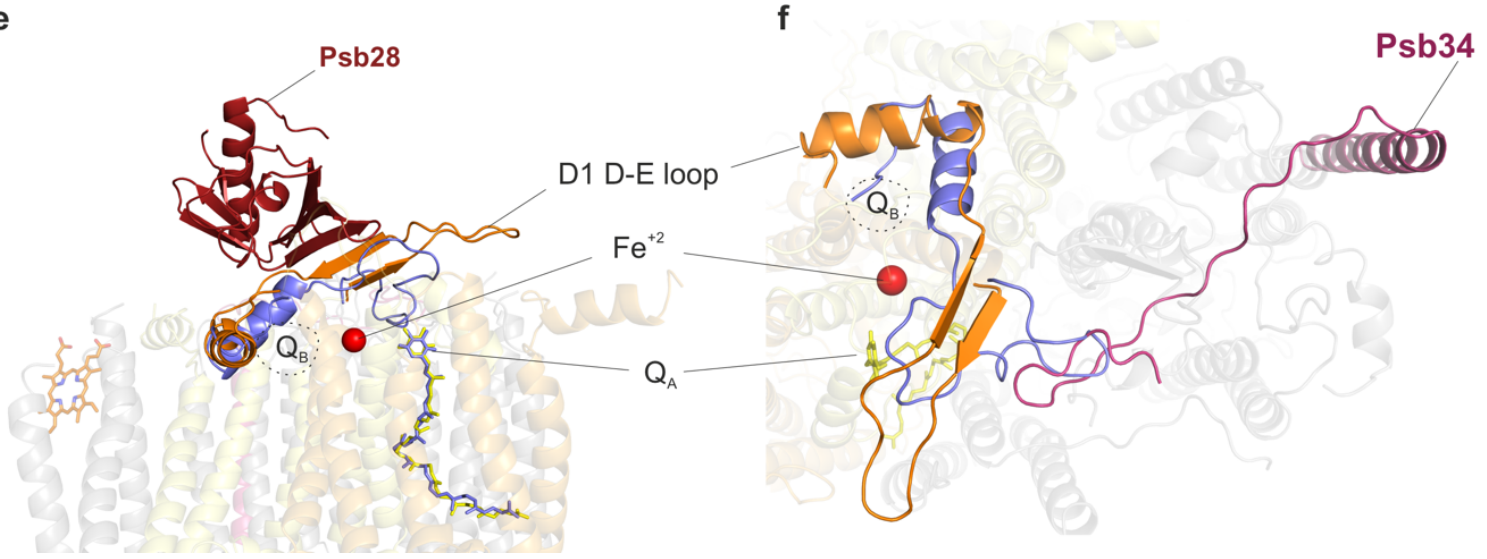

Fig. 4. Structural changes of the D1 and D2 D-E loops induced by binding of Psb28 and Psb34. a, Side view of the CP43 antenna protein in PSII-I (teal) and the PSII-M control (light blue). b. Structural changes between PSII-I and the PSII-M control in the cytoplasmic D2 D-E loop (yellow: PSII-I, blue: PSII-M) and attachment of CP43 (teal: PSII-I, light blue: PSII-M control) (top view). Details of the structural changes in the D2 loop are shown in Fig. S5A and B. c, Side view and $\mathbf{d}$, top view of the PSII-I structure (orange) compared to the PSII-M control (light blue) and mature monomeric PSII (light red, PDB-ID 3KZI). e, Side view and f, top view of the Psb28-induced structural changes in the D1 D-E loop (orange) and perturbation of the $\mathrm{Q}_{в}$ binding site compared to PSII-M (light blue), which lacks the assembly factors. QA is shown in yellow (PSII-I) or light blue (PSII-M), respectively. See Fig. S5c-h for enlarged views of the $\mathrm{Q}_{\mathrm{A}}$ and $\mathrm{Q}_{B}$ binding site and the adjacent non-heme iron. 


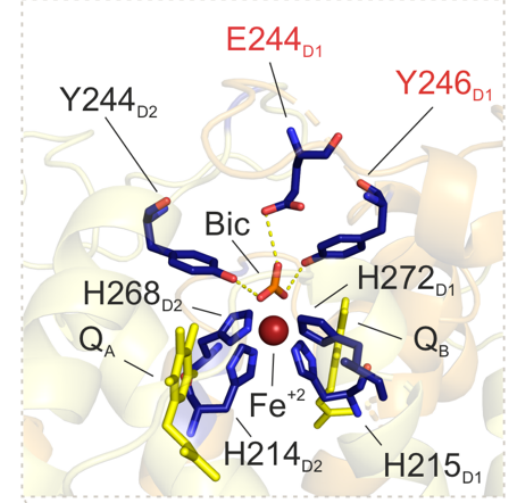

c
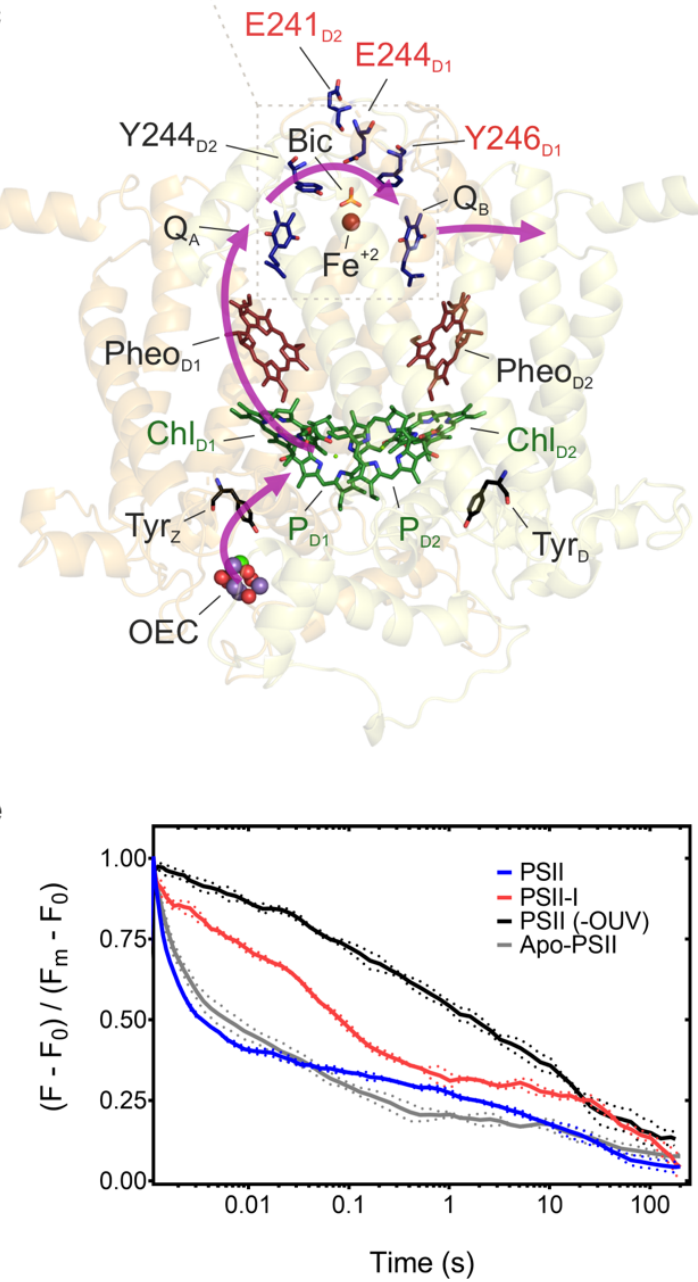

b

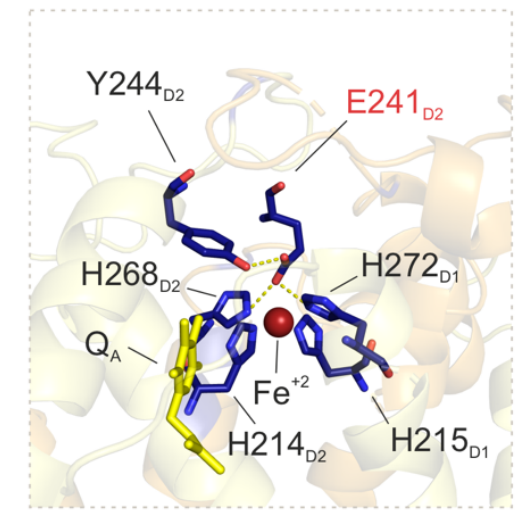

d

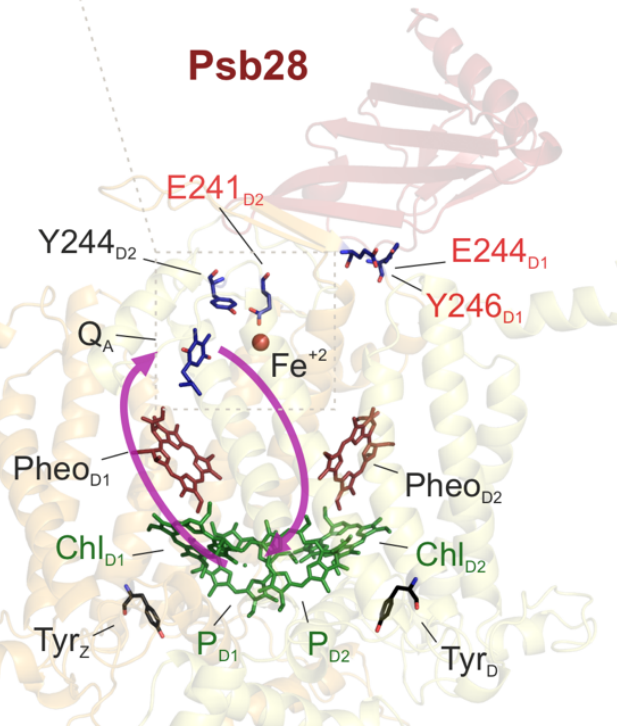

f

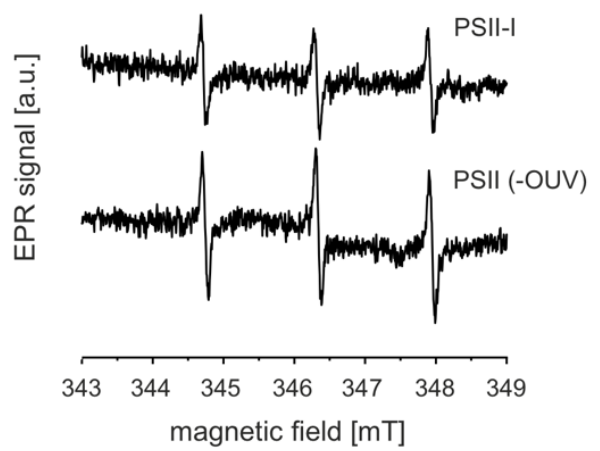

Fig. 5: Binding of Psb28 displaces bicarbonate as a ligand of the non-heme iron and protects PSII from damage. a, The electron transfer from $\mathrm{PQ}_{\mathrm{A}}$ to $\mathrm{PQ}_{\mathrm{B}}$ is coordinated by the non-heme iron $\left(\mathrm{Fe}^{2+}\right)$, with the binding of bicarbonate (Bic) serving as a regulatory mechanism ${ }^{57}$ in mature PSII (PDB-ID 3 WU2). b, Binding of Psb28 to the PSII-I assembly intermediate induces a conformational change in the cytoplasmic D2 D-E loop, where the side 
chain of Glu241 replaces bicarbonate as a ligand of the non-heme iron. The respective fits of the non-heme iron binding sites are shown in Fig. S5E and F. A similar coordination is also found in non-oxygenic bacterial reaction centers $^{69}$ (Fig. S6C). c, Electron transfer (purple arrows) in mature PSII. Light-induced charge separation at the reaction center chlorophylls $\left(\mathrm{P}_{\mathrm{D} 1}, \mathrm{PD}_{\mathrm{D} 2}, \mathrm{Ch}_{\mathrm{D} 1}, \mathrm{Ch}_{\mathrm{D} 2}\right)$ leads to electron transfer via pheophytin (PheoD1) and plastoquinone $\mathrm{A}\left(\mathrm{Q}_{\mathrm{A}}\right)$ towards $\mathrm{Q}_{\mathrm{B}}$. The electron gap at the reaction center is filled by the oxygen evolving complex (OEC). d, Reoxidation of $\mathrm{Q}_{A}{ }^{-}$by direct and safe charge recombination is favored in the PSII assembly intermediate, as indicated by the purple arrows. e, Flash-induced fluorescence decay of PSII. Blue lines represent active PSII and red lines correspond to PSII-I. Black and grey lines represent PSII control samples without a functional OEC (Apo-PSII: hydroxylamine treated PSII; PSII (-OUV): extrinsic proteins removed). Dotted corridors depict SD ( $n$ =3). $\mathbf{f}$, The protective role of Psb28 binding was further confirmed by EPR spectroscopy using the spin probe TEMPD, which is specific for ${ }^{1} \mathrm{O}_{2}$, the major reactive oxygen species in PSII generated by triplet chlorophyll $\left({ }^{3} \mathrm{P}\right)$. 


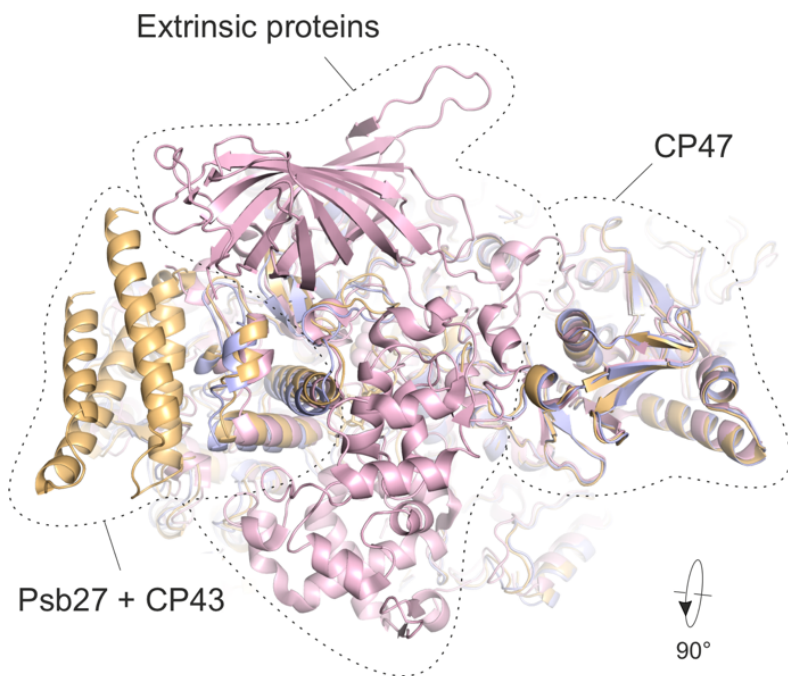

C

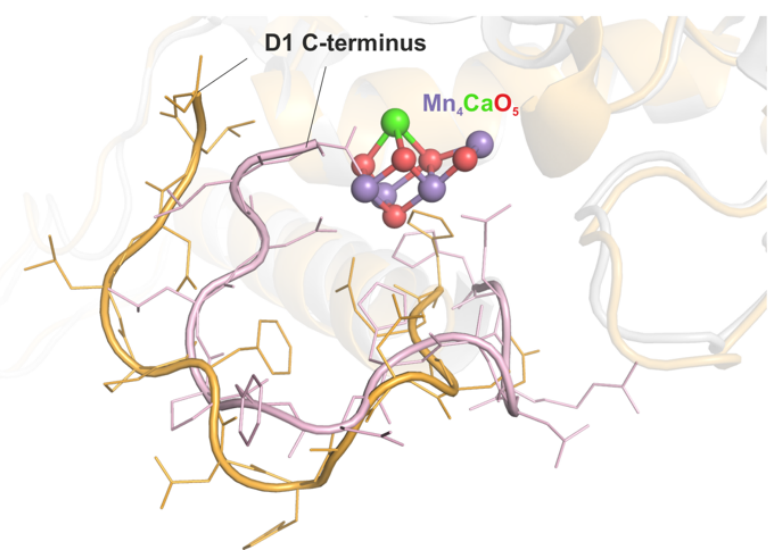

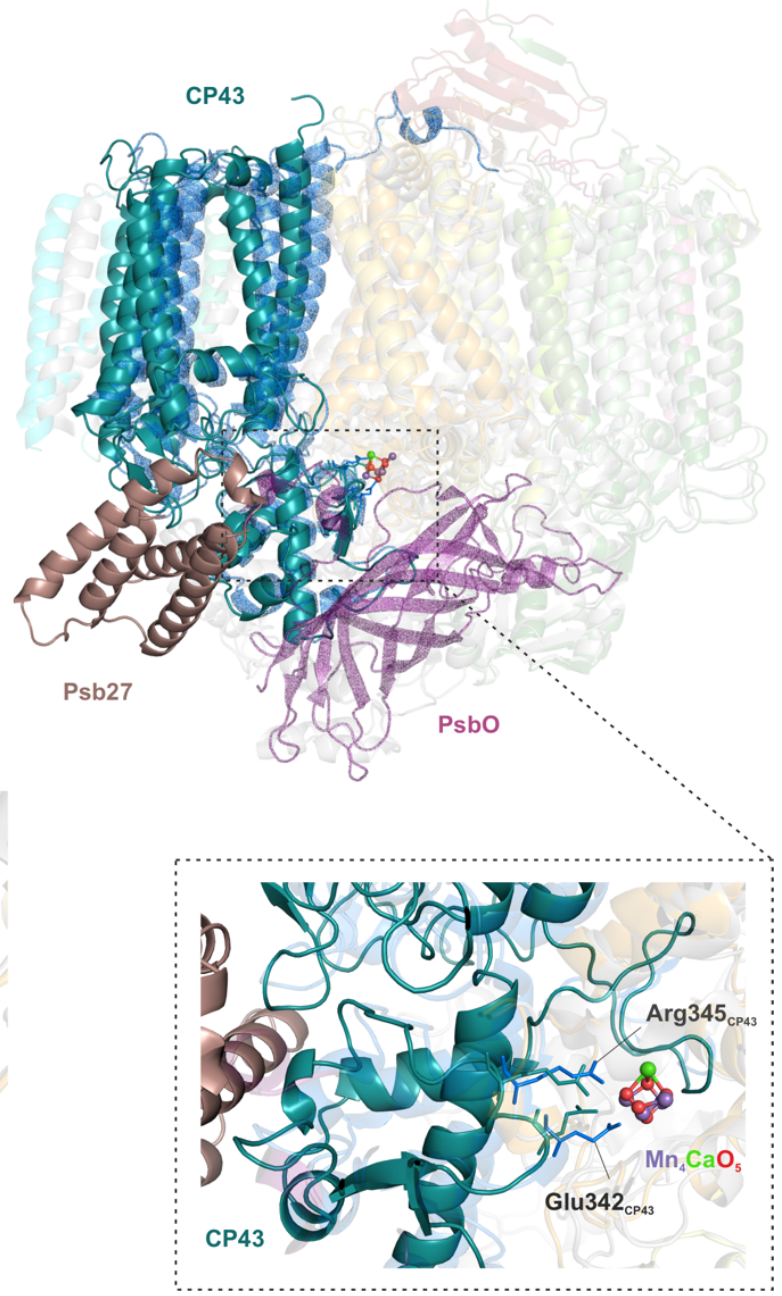

Fig. 6: The role of Psb27 in $\mathrm{Mn}_{4} \mathrm{CaO}_{5}$ cluster assembly. a, Bottom view of the luminal PSII surface for PSII-I (orange), the PSII-M control (light blue) and mature monomeric PSII (PDB-ID 3KZI) (light red). b, Side view of CP43 (teal) and Psb27 (brown) in PSII-I, as well as of CP43 (blue) and PsbO (purple) in mature monomeric PSII (PDB-ID 3KZI). Dashed box: CP43 E loop with residues Arg345 and Glu342 (shown as sticks), which form the second coordination sphere of the $\mathrm{Mn}_{4} \mathrm{CaO}_{5}$ cluster. We changed the numbering of $\mathrm{CP} 43$ residues due to a corrected N-terminal sequence (www.UniProt.org). The residues correspond to Arg357 and Glu354 in previous publications. The high-resolution structure of the $\mathrm{Mn}_{4} \mathrm{CaO}_{5}$ cluster is taken from Umena et al. 2011 (PDB-ID 3WU2). c, Position of the D1 C-terminus in PSII-I (orange) and mature monomeric PSII (PDB-ID 3KZI) (light red). 
a

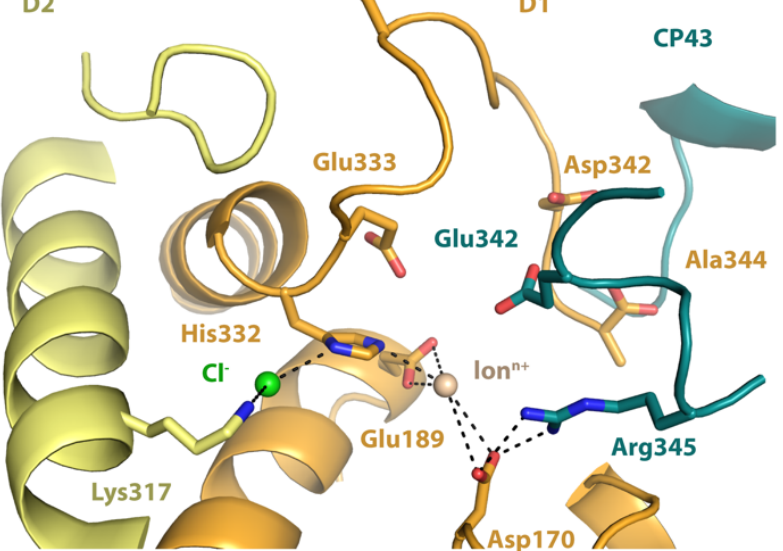

c $\mathrm{D} 2$
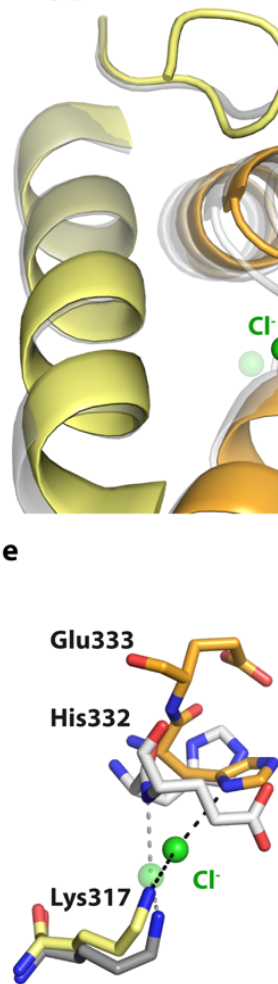

D2

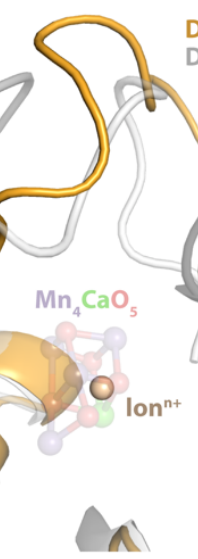

f

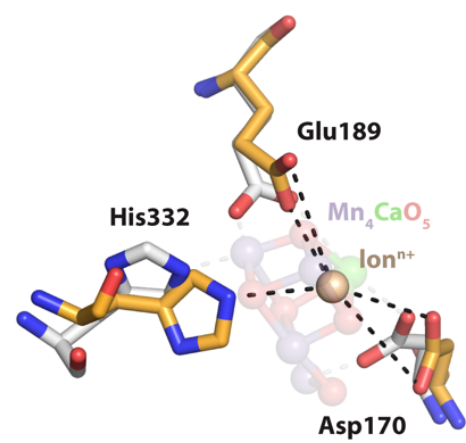

b

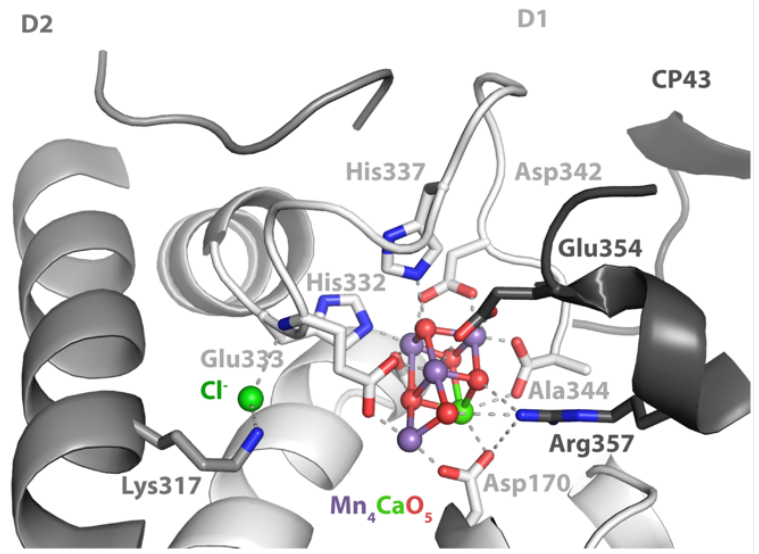

d

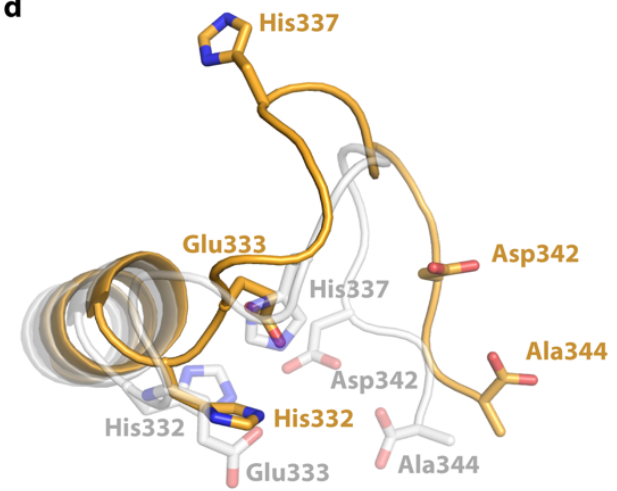

g
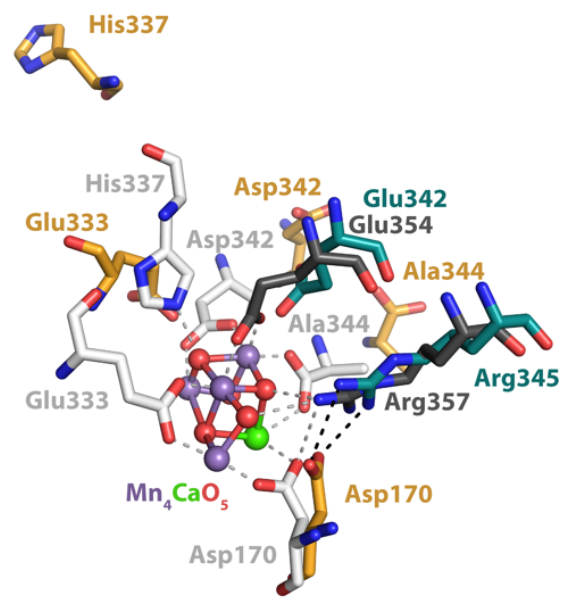

Figure 7: Conformational changes within the active site of the $\mathrm{Mn}_{4} \mathrm{CaO}_{5}$ cluster. The $\mathrm{Mn}_{4} \mathrm{CaO}_{5}$ cluster performs PSII's unique water-splitting reaction. a, The active site of the $\mathrm{Mn}_{4} \mathrm{CaO}_{5}$ cluster is resolved within our PSII-I structural model but is not yet oxygen-evolving. b, Crystal structure of the oxygen-evolving, mature PSII (PDB-ID 3WU2, resolution 1.9 Å). c, Overlay of both structures, illustrating significant differences in the backbone conformation of the D1 and D2 C-terminal tails. d, Accompanying side chain rearrangements of the D1 Cterminus. The $\mathrm{Cl}^{-}(\mathbf{e}), \mathrm{Ion}^{+}(\mathbf{f})$ and $\mathrm{Mn}_{4} \mathrm{CaO}_{5}$ (g) cluster coordination partners are compared in detailed. The validation of the fit to density for the structural details shown here is provided in Figure S7. 